\title{
Oligomers as Intermediates in Ring-Closing Metathesis
}

\author{
Jay C. Conrad, Melanie D. Eelman, João A. Duarte Silva, Sebastien Monfette, Henrietta H. \\ Parnas, Jennifer L. Snelgrove and Deryn E. Fogg* \\ Center for Catalysis Research and Innovation, Department of Chemistry, University of Ottawa, \\ 10 Marie Curie, Ottawa ON, Canada, K1N 6N5
}

\section{SUPPORTING INFORMATION}

\section{Contents:}

1. Experimental procedures.

2. Preparation and characterization of dienes and RCM products.

3. References.

*Corresponding author. E-mail: dfogg@uottawa.ca. Fax: (613) 562-5170 


\section{Experimental Procedures.}

General Procedures. Synthetic procedures were carried out at room temperature $\left(22{ }^{\circ} \mathrm{C}\right)$ under $\mathrm{N}_{2}$ or Ar, using standard Schlenk or drybox techniques. Dry, oxygen-free solvents were obtained using a Glass Contour or Anhydrous Engineering solvent purification system, and stored over Linde $4 \AA$ molecular sieves. $\mathrm{CDCl}_{3}$ was distilled from $\mathrm{CaH}_{2}$, then degassed by consecutive freeze/pump/thaw cycles, and stored over activated sieves. $\mathrm{C}_{6} \mathrm{D}_{6}$ was dried over activated sieves (Linde $4 \AA$ ) and degassed by consecutive freeze/pump/thaw cycles. Substrate $\mathbf{8 b}$ was obtained from Aldrich Chemicals. Catalysts $\mathbf{1}^{1}$ and $\mathbf{2},{ }^{2}$ and substrates $4 \mathbf{a},{ }^{3} \mathbf{4 b},{ }^{3} \mathbf{4 c},{ }^{4} \mathbf{1 0 b},{ }^{5}$ and $\mathbf{1 4}^{6}$ were prepared according to literature procedures. Chromatographic purification of RCM products was carried out in air. Characterization data for $\mathbf{5 a},{ }^{3} \mathbf{5 b},{ }^{3} \mathbf{5 c},{ }^{4} \mathbf{1 1} \mathbf{b}^{5}$ and $\mathbf{1 5}^{6}$ agreed with literature values. All substrates were distilled under vacuum and degassed by consecutive freeze/pump/thaw cycles. ${ }^{1} \mathrm{H}$ NMR $(300,400$ or $500 \mathrm{MHz})$ and ${ }^{13} \mathrm{C}$ NMR $(75$ or $125 \mathrm{MHz})$ spectra were recorded on Bruker Avance-300, Bruker Avance-400 or Bruker Avance-500 spectrometers. IR spectra were recorded on thin films of neat oils ( $\mathrm{NaCl}$ plates) using a Bomem MB100 IR spectrometer. GC-FID analyses were performed on an Agilent 6890 Series gas chromatograph equipped with an FID detector and a Varian fused silica CP WAX 52CB column. Samples were analyzed immediately upon removal of aliquots from catalytic runs. Identity and retention times for all dienes and RCM products were confirmed by comparison with authentic materials, and by GC-MS, NMR, and HR-MS analysis. Reaction rates were determined by GCFID (from both consumption of starting material and formation of product; proportion of ADMET oligomers determined by difference; maximum $1 \%$ isomerization). Peak areas were referenced against THN (1,2,3,4-tetrahydronaphthalene) as internal standard, interpolating from 
calibration curves covering the concentration regime to be monitored. Agreement between replicate runs was within $\pm 3 \%$. MALDI-TOF MS analysis was performed using a Bruker OmniFlex MALDI-TOF mass spectrometer equipped with a nitrogen laser (337 $\mathrm{nm})$, and interfaced to an MBraun glovebox. Data were collected in positive reflectron mode, with the accelerating voltage held at $20 \mathrm{kV}$. Matrix (pyrene) and analyte solutions were prepared in $\mathrm{CH}_{2} \mathrm{Cl}_{2}$ at concentrations of $20 \mathrm{mg} / \mathrm{mL}$ and $1 \mathrm{mg} / \mathrm{mL}$, respectively. Samples were mixed in a matrix:analyte ratio of 20:1 (v/v).

\section{Representative Procedures for Ring-Closing Metathesis Reactions.}

(a) RCM (Ziegler method). A three-neck round-bottom flask was fitted with a condenser and two addition funnels. Solutions of catalyst $1(13 \mathrm{mg}, 15 \mu \mathrm{mol})$ and substrate $\mathbf{4 a}(80 \mathrm{mg}, 0.30$ mmol, with $10 \mathrm{mg}(0.076 \mathrm{mmol}) \mathrm{THN}$ as internal standard), each in $20 \mathrm{~mL}$ solvent, were independently added dropwise over 15 minutes to $20 \mathrm{~mL}$ of refluxing $\mathrm{CH}_{2} \mathrm{Cl}_{2}$ under a flow of argon. A $100 \mu \mathrm{L}$ sample of the diene solution was removed and diluted to $5 \mathrm{mM}$ to establish the $t_{\mathrm{o}}$ reading prior to reaction. Aliquots were removed periodically for GC analysis, beginning at 15 minutes, using a gas-tight syringe. GC retention times and integrations were confirmed with samples of authentic material, for which response factors were independently measured. RCM of 4a using 1 was complete in $9 \mathrm{~h}$.

(b) General RCM reactions (single-dose addition; $100 \mathbf{m M}$ ). A $20 \mathrm{~mL}$ scintillation vial was charged with 4a (266 mg, $0.998 \mathrm{mmol})$, THN internal standard $(66 \mathrm{mg}, 0.50 \mathrm{mmol})$ and $\mathrm{CH}_{2} \mathrm{Cl}_{2}$ $(10 \mathrm{~mL})$. A $10 \mu \mathrm{L}$ aliquot was removed as the sample corresponding to $t_{0}$, diluted to $5 \mathrm{mM}$, and subjected to GC analysis. Catalyst $1(42 \mathrm{mg}, 0.05 \mathrm{mmol})$ was added to the reaction solution, which was stirred rapidly in the open vessel. Six $1.5 \mathrm{~mL}$ aliquots of this solution were transferred 
to six independent, $4 \mathrm{~mL}$ vials, which were stirred while open to the glovebox atmosphere, to promote loss of ethylene. After 15 minutes (at which time consumption of diene 4 a was complete), the daughter vials were sealed with PTFE-lined screw caps, brought out of the glovebox, and inserted in an aluminium block, in which the solutions were heated to reflux. Analysis was carried out at fixed time intervals $(15,30$, and $45 \mathrm{~min} ; 1,1.5$, and $2 \mathrm{~h}$ ), by removing a vial from the block, allowing it to cool to room temperature, and removing an aliquot. This was diluted to $5 \mathrm{mM}\left(50 \mu \mathrm{L}\right.$ in $\left.950 \mu \mathrm{L} \mathrm{CH}_{2} \mathrm{Cl}_{2}\right)$, and immediately subjected to GC analysis. By 30 minutes, yields of 5a plateau at 10\%, showing no further change over $5 \mathrm{~h}$.

(c) General RCM reactions (single-dose addition; $5 \mathrm{mM}$ ). The procedure was carried out as in (b), using $20 \mathrm{mg} \mathrm{4a}(0.075 \mathrm{mmol}), 10 \mathrm{mg}$ internal standard THN (0.076 mmol), and $15 \mathrm{~mL}$ $\mathrm{CH}_{2} \mathrm{Cl}_{2}$. A $1 \mathrm{~mL}$ aliquot was removed for $\mathrm{GC}$ analysis, after which $295 \mu \mathrm{L}$ of 1 (5.50 $\mu \mathrm{mol}, 5$ mol\%) of a stock solution (10 $\mathrm{mg}$ in $1.00 \mathrm{~mL} ; 11.8 \mathrm{mM})$ was added. The reaction was stirred vigorously in an open vessel, and $1 \mathrm{~mL}$ samples were distributed to eight 4-mL (open) screw-top vials, and treated as in (b). Samples were analyzed without further dilution, at the same time intervals as in (b). RCM of $\mathbf{4 a}$ was complete in $5 \mathrm{~h}$; for other substrates, see Table 1 (paper).

(d) RCM of 4a (single-dose addition; initially $100 \mathrm{mM}$, then diluted to $5 \mathrm{mM}$ ). The procedure was carried out as in (b) using $40 \mathrm{mg} \mathbf{4 a}$, but at 15 minutes, the sample was diluted to $5 \mathrm{mM}$. Subsequent procedures were carried out as in (c). RCM of $\mathbf{4 a}$ was complete in $1 \mathrm{~h}$.

MALDI-TOF MS Analysis of ADMET Oligomer for 4a. The oligomer was prepared by the Ziegler method (see (a) above), without internal standard, by arresting the reaction after the 15 minute addition period. The solvent was stripped off to yield a greasy solid, which was subjected to MALDI-TOF MS analysis without purification. The full spectrum is shown in Figure S1. 


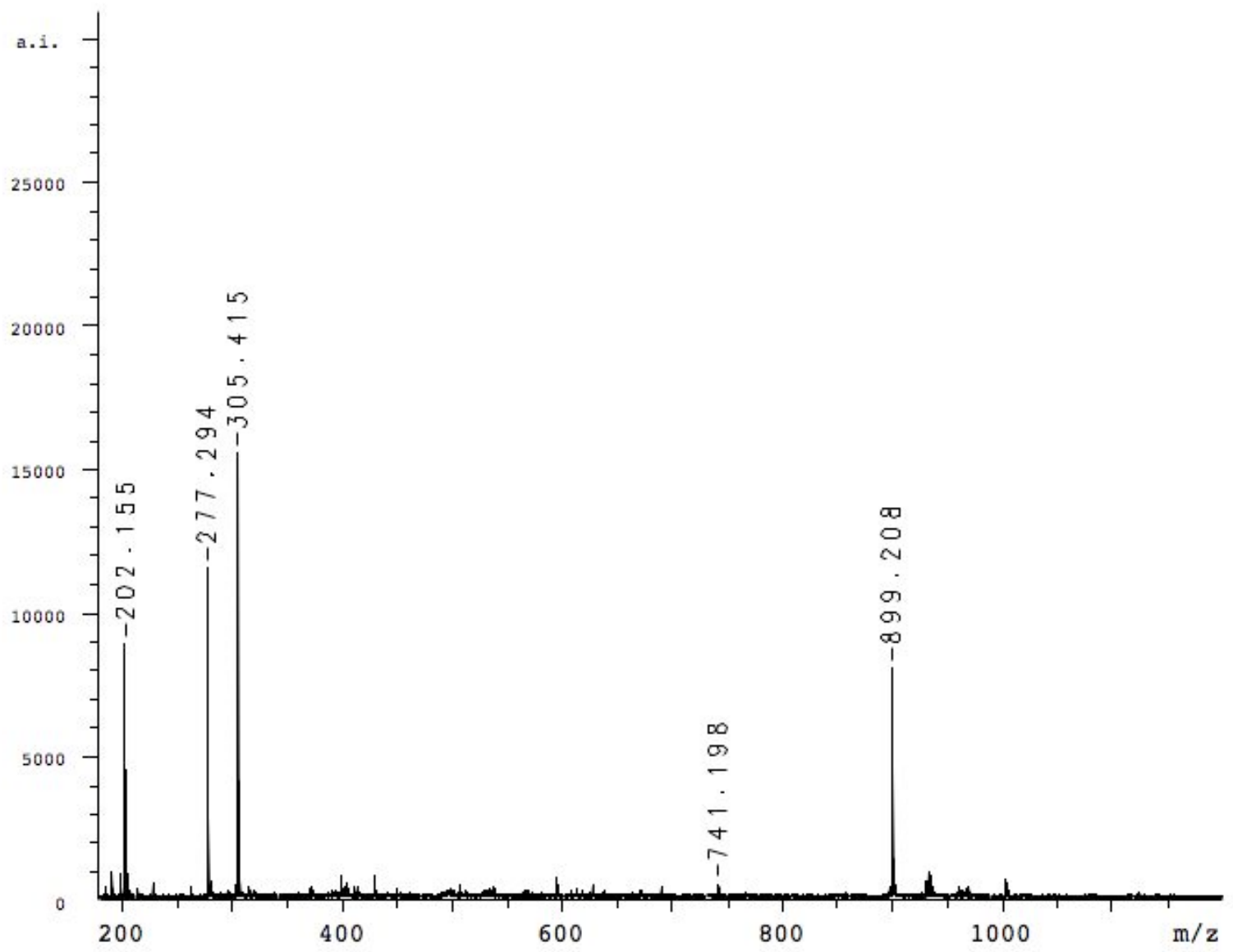

Figure S1. Full-range MALDI-TOF mass spectrum of the ADMET oligomer of 4a (pyrene matrix; $m / z$ 202.1). Signal at 305.4 due to [IMes+H] $]^{+}$. 


\section{Preparation and characterization of dienes and RCM products.}

\subsection{Preparation and characterization of dienes.}

Preparation of 6 a.

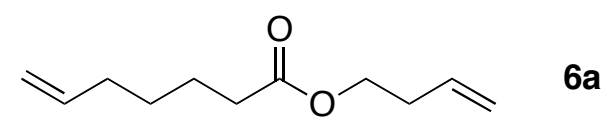

The procedure used for $\mathbf{4 a}$ was followed, using 6-heptanoic acid (2.01 g, $16 \mathrm{mmol})$ and 3-butene1-ol (1.05 equiv). Yield 1.26 g (44\%). ${ }^{1} \mathrm{H}$ NMR $\left(\mathrm{CDCl}_{3}, 300 \mathrm{MHz}\right): \delta$ 5.86-5.69 (m, 2H, $\left.\mathrm{CH}\right)$, 5.14-4.89 (m, 4H, =CH $), 4.11\left(\mathrm{t},{ }^{3} \mathrm{~J}_{\mathrm{HH}}=6.7 \mathrm{~Hz}, 2 \mathrm{H}, \mathrm{OCH}_{2}\right), 2.41-2.25\left(\mathrm{~m}, 4 \mathrm{H}, \mathrm{CH}_{2}\right), 2.10-2.00$ (m, 2H, $\left.\mathrm{CH}_{2}\right), 1.69-1.56\left(\mathrm{~m}, 2 \mathrm{H}, \mathrm{CH}_{2}\right), 1.46-1.34\left(\mathrm{~m}, 2 \mathrm{H}, \mathrm{CH}_{2}\right) .{ }^{13} \mathrm{C}\left\{{ }^{1} \mathrm{H}\right\}\left(\mathrm{CDCl}_{3}, 125 \mathrm{MHz}\right)$ : $\delta 173.6,138.3,134.0,117.1,114.6,63.2,34.1,33.3,33.1,28.3,24.4 . \mathrm{IR} v\left(\mathrm{~cm}^{-1}\right) 3079(\mathrm{~s}), 2932$ (s), 2860 (s), 1736 (s), 1642 (s), 1457 (m), 1418 (m), 1387 (m), 1234 (s), 1171 (s), 992 (m), 913 (s), $741(w), 636(w)$. HR-MS (+EI): Calc'd for $\mathrm{C}_{7} \mathrm{H}_{11} \mathrm{O},\left[\mathrm{M}-\mathrm{C}_{4} \mathrm{H}_{7} \mathrm{O}\right]^{+\bullet}, m / z$ 111.0810. Found, $m / z$ 111.0808 .

\section{Preparation of $6 \mathbf{b}$.}

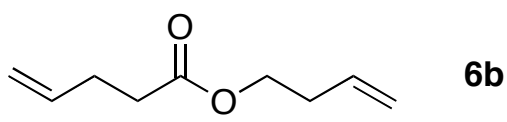

The procedure used for 4a was followed, using 4-pentenoic acid (4.04 g, $40.4 \mathrm{mmol}$ ) and 3butene-1-ol (3.06 g, $42.4 \mathrm{mmol})$. Yield $3.6 \mathrm{~g}$ (58\%). ${ }^{1} \mathrm{H}$ NMR $\left(\mathrm{CDCl}_{3}, 300 \mathrm{MHz}\right)$ : $\delta$ 5.83-5.71 $(\mathrm{m}, 2 \mathrm{H}, \mathrm{CH}), 5.09-4.95\left(\mathrm{~m}, 4 \mathrm{H},=\mathrm{CH}_{2}\right), 4.10\left(\mathrm{t},{ }^{3} J_{\mathrm{HH}}=5.5 \mathrm{~Hz}, 2 \mathrm{H}, \mathrm{OCH}_{2}\right), 2.39-2.33(\mathrm{~m}, 6 \mathrm{H}$, $\left.\mathrm{CH}_{2}\right) .{ }^{13} \mathrm{C}\left\{{ }^{1} \mathrm{H}\right\}\left(\mathrm{CDCl}_{3}, 125 \mathrm{MHz}\right): \delta 172.8,136.6,133.9,117.1,115.3,63.3,33.4,33.0,28.8 . \mathrm{IR}$ $v\left(\mathrm{~cm}^{-1}\right) 3081(\mathrm{~s}), 2981(\mathrm{~s}), 2921(\mathrm{~s}), 1735(\mathrm{~s}), 1642(\mathrm{~s}), 1420(\mathrm{~s}), 1386(\mathrm{~m}), 1352(\mathrm{~m}), 1246(\mathrm{~s})$, 1177 (s), 990 (s), 916 (s), 787 (w), 637 (w). HR-MS (+EI): Calc'd for $\mathrm{C}_{6} \mathrm{H}_{9} \mathrm{O}_{2},\left[\mathrm{M}-\mathrm{C}_{3} \mathrm{H}_{5}\right]^{+\bullet}, m / z$ 113.0603. Found, $m / z 113.0603$. 
Preparation of $6 c$.

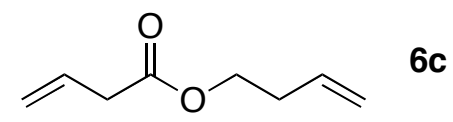

The compound was prepared as for $\mathbf{4 a}$, rather than by the reported method, ${ }^{7}$ using 3-butenoic acid (5.3 g, $61.6 \mathrm{mmol})$ and 3-buten-1-ol (4.7 g, $65.2 \mathrm{mmol})$. Yield $4.6 \mathrm{~g}(53 \%) .{ }^{1} \mathrm{H}$ NMR $\left(\mathrm{CDCl}_{3}, 300 \mathrm{MHz}\right): \delta \quad 5.97-5.69(\mathrm{~m}, 2 \mathrm{H}, \mathrm{CH}), 5.18-5.03\left(\mathrm{~m}, 4 \mathrm{H},=\mathrm{CH}_{2}\right), 4.13\left(\mathrm{t},{ }^{3} J_{\mathrm{HH}}=6.6 \mathrm{~Hz}\right.$, $\left.2 \mathrm{H}, \mathrm{OCH}_{2}\right), 3.07\left(\mathrm{dt},{ }^{3} J_{\mathrm{HH}}=6.9 \mathrm{~Hz},{ }^{4} J_{\mathrm{HH}}=1.5 \mathrm{~Hz}, 2 \mathrm{H}, \mathrm{CH}_{2}\right), 2.37\left(\mathrm{qt},{ }^{3} J_{\mathrm{HH}}=6.9 \mathrm{~Hz},{ }^{4} J_{\mathrm{HH}}=1.5\right.$ $\left.\mathrm{Hz}, 2 \mathrm{H}, \mathrm{CH}_{2}\right) .{ }^{13} \mathrm{C}\left\{{ }^{1} \mathrm{H}\right\}$ NMR $\left(\mathrm{CDCl}_{3}, 75 \mathrm{MHz}\right): \delta 171.4,133.8,130.2,118.4,117.2,63.6,39.1$, 33.0. IR v (cm $\left.\mathrm{cm}^{-1}\right) 3082(\mathrm{w}), 2983(\mathrm{w}), 2960$ (w), 2905 (w), 1740 (s), 1643 (s), 1426 (w), 1328 (m), 1252 (m), 1173 (s), 992 (m), 919 (s). HR-MS (+EI): Calc'd for $\mathrm{C}_{5} \mathrm{H}_{7} \mathrm{O}_{2},\left[\mathrm{M}-\mathrm{C}_{3} \mathrm{H}_{5}\right]^{+\bullet}, \mathrm{m} / \mathrm{z}$ 99.0446. Found, $m / z 99.0445$.

\section{Preparation of 8a.}

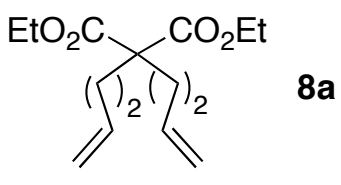

To a solution of sodium ethoxide $(2.69 \mathrm{~g}, 39.51 \mathrm{mmol})$ in dry DMF $(20 \mathrm{~mL})$ was added diethyl malonate $(2.11 \mathrm{~g}, 13.17 \mathrm{mmol}, 2.0 \mathrm{~mL})$. The mixture was stirred for $9 \mathrm{~h}$ under $\mathrm{N}_{2}$, after which 4 bromo-1-butene $(944 \mathrm{mg}, 6.99 \mathrm{mmol}$ ) was added, and the reaction was stirred for 17 hours further. The mono-allyl adduct was isolated by quenching with $5 \% \mathrm{HCl}(25 \mathrm{~mL})$ and extracting with $\mathrm{Et}_{2} \mathrm{O}(4 \times 20 \mathrm{~mL})$. The organic phase was dried (brine, $\left.\mathrm{MgSO}_{4}\right)$, filtered, and stripped of solvent to give a brown oil, which was purified by chromatography on silica gel (10\% EtOAc/hexanes). To a solution of the mono-allyl adduct $(1.25 \mathrm{~g}, 5.84 \mathrm{mmol})$ in dry DMF (10 $\mathrm{mL}$ ) was added solid $\mathrm{NaH}(280 \mathrm{mg}, 11.6 \mathrm{mmol})$. The mixture was stirred for $1 \mathrm{~h}$, after which $4-$ bromo-1-butene (945 mg, $6.99 \mathrm{mmol}$ ) was added, and stirring continued at $80{ }^{\circ} \mathrm{C}$ for $20 \mathrm{~h}$ under 
$\mathrm{N}_{2}$. The solution was worked up as above and purified by distillation. Yield: $397 \mathrm{mg}, 25 \% .{ }^{1} \mathrm{H}$ $\operatorname{NMR}\left(\mathrm{CDCl}_{3}, 300 \mathrm{MHz}\right): \delta 5.85-5.70(\mathrm{~m}, 2 \mathrm{H}, \mathrm{CH}), 5.08-4.92\left(\mathrm{~m}, 4 \mathrm{H},=\mathrm{CH}_{2}\right), 4.20\left(\mathrm{q},{ }^{3} J_{\mathrm{HH}}=\right.$

$\left.7.1 \mathrm{~Hz}, 4 \mathrm{H}, \mathrm{CO}_{2} \mathrm{CH}_{2}\right), 2.04-1.90\left(\mathrm{~m}, 8 \mathrm{H}, \mathrm{CH}_{2} \mathrm{CH}_{2}\right), 1.27\left(\mathrm{t},{ }^{3} \mathrm{~J}_{\mathrm{HH}}=7.1 \mathrm{~Hz}, 6 \mathrm{H}, \mathrm{CH}_{3}\right) .{ }^{13} \mathrm{C}\left\{{ }^{1} \mathrm{H}\right\}$ NMR (CDCl $3,75 \mathrm{MHz}): \delta 171.9,138.0,115.4,61.5,57.4,31.9,28.8,14.5 . \mathrm{IR} v\left(\mathrm{~cm}^{-1}\right) 3079(\mathrm{~m})$, 2980 (s), 2940 (s), 1731 (s), 1642 (m), 1448 (m), 1366 (m), 1296 (m), 1263 (s), 1205 (s), 1145 (s), 1096 (m), 1031 (s), 994 (m), 913 (s), 861 (s), 731 (s). HR-MS (+EI): Calc'd for $\mathrm{C}_{13} \mathrm{H}_{19} \mathrm{O}_{3}$, $\left[\mathrm{M}-\mathrm{C}_{2} \mathrm{H}_{5} \mathrm{O}\right]^{+\bullet}, m / z$ 223.1334. Found, $m / z$ 223.1352.

Preparation of 10a.

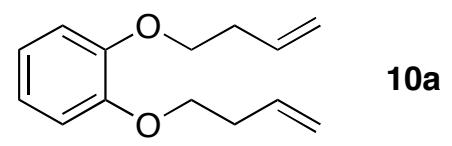

The procedure used for $\mathbf{1 0 b}$ was followed, using catechol (1.00 g, $9.08 \mathrm{mmol})$, NaH (240 mg, $9.99 \mathrm{mmol})$ and 1-bromo-3-butene (1.49 g, $9.99 \mathrm{mmol})$. Yield: $425 \mathrm{mg}(34 \%) .{ }^{1} \mathrm{H} \mathrm{NMR}\left(\mathrm{CDCl}_{3}\right.$, $400 \mathrm{MHz}): \delta 6.93(\mathrm{~s}, 4 \mathrm{H}, \mathrm{ArH}), 6.05-5.87(\mathrm{~m}, 2 \mathrm{H}, \mathrm{CH}), 5.19-5.08\left(\mathrm{~m}, 4 \mathrm{H},=\mathrm{CH}_{2}\right), 4.07\left(\mathrm{t},{ }^{3} J_{\mathrm{HH}}=\right.$ $\left.6.8 \mathrm{~Hz}, 4 \mathrm{H}, \mathrm{OCH}_{2}\right), 2.73-2.45\left(\mathrm{~m}, 4 \mathrm{H}, \mathrm{OCH}_{2} \mathrm{CH}_{2}\right) .{ }^{13} \mathrm{C}\left\{{ }^{1} \mathrm{H}\right\} \mathrm{NMR}\left(\mathrm{CDCl}_{3}, 75 \mathrm{MHz}\right): \delta 149.0$, 134.6, 121.3, 116.9, 114.5, 68.6, 33.8. IR $v\left(\mathrm{~cm}^{-1}\right) 3087(\mathrm{~m}), 2978(\mathrm{~m}), 2925(\mathrm{~s}), 2872(\mathrm{~m}), 1732$ (w), 1644 (w), $1591(\mathrm{~m}), 1054$ (s), 1466 (m), 1450 (m), 1382 (m), 1249 (s), 1218 (s), 1120 (s), 1028 (s), 987 (m), 918 (m), 739 (s). HR-MS (+EI): Calc'd for $\mathrm{C}_{14} \mathrm{H}_{18} \mathrm{O}_{2}, \mathrm{M}^{+\bullet}, \mathrm{m} / z$ 218.1307. Found, $m / z 218.1304$. 


\section{Preparation of 12a.}

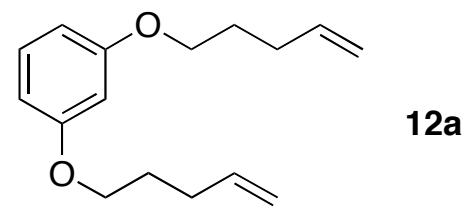

The procedure used for $10 \mathrm{~b}$ was followed, using resorcinol $(1.0 \mathrm{~g}, 9.08 \mathrm{mmol}), \mathrm{NaH}(240 \mathrm{mg}$, $9.99 \mathrm{mmol})$ and 1-bromo-4-pentene (1.49 g, $9.99 \mathrm{mmol})$. Yield: $637 \mathrm{mg}(29 \%)$, with $349 \mathrm{mg}$ (22\%) of mono-substituted material. ${ }^{1} \mathrm{H}$ NMR $\left(\mathrm{CDCl}_{3}, 400 \mathrm{MHz}\right): \delta 7.16\left(\mathrm{t},{ }^{3} J_{\mathrm{HH}}=8.1 \mathrm{~Hz}, 1 \mathrm{H}\right.$, $\operatorname{Ar} H), 6.56-6.42(\mathrm{~m}, 3 \mathrm{H}, \mathrm{Ar} H), 5.95-5.78(\mathrm{~m}, 2 \mathrm{H}, \mathrm{CH}), 5.14-4.96\left(\mathrm{~m}, 4 \mathrm{H},=\mathrm{CH}_{2}\right), 3.95\left(\mathrm{t},{ }^{3} \mathrm{~J}_{\mathrm{HH}}=\right.$ $\left.9.1 \mathrm{~Hz}, 4 \mathrm{H}, \mathrm{OCH}_{2}\right), 2.33-2.19\left(\mathrm{~m}, 4 \mathrm{H}, \mathrm{CH}_{2}\right), 1.96-1.81\left(\mathrm{~m}, 4 \mathrm{H}, \mathrm{CH}_{2}\right) \cdot{ }^{13} \mathrm{C}\left\{{ }^{1} \mathrm{H}\right\} \mathrm{NMR}\left(\mathrm{CDCl}_{3}, 75\right.$ MHz): $\delta 160.2,137.8,129.8,115.2,106.6,101.4,67.1,30.1,28.4 . \operatorname{IR} v\left(\mathrm{~cm}^{-1}\right) 3077$ (s), $2983(\mathrm{~s})$, 2938 (s), 2872 (s), 1640 (s), 1590 (s), 1492 (s), 1468 (s), 1387 (s), 1333 (s), 1286 (s), 1264 (m), 1181 (s), 1154 (s), 1050 (s), 994 (m), 913 (s), 834 (s), 761 (s), 686 (s). HR-MS (+EI): Calc'd for $\mathrm{C}_{16} \mathrm{H}_{22} \mathrm{O}_{2}, \mathrm{M}^{+\bullet}, m / z$ 246.1620. Found, 246.1614.

Preparation of 12b.

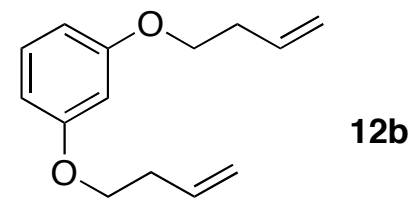

The procedure used for $\mathbf{1 0 b}$ was followed, using resorcinol (530 mg, $4.813 \mathrm{mmol}$ ), NaH (139 $\mathrm{mg}, 5.776 \mathrm{mmol}$ ) and 1-bromo-3-butene (663 mg, $4.910 \mathrm{mmol}, 0.500 \mathrm{~mL})$. Yield $94 \mathrm{mg}(9 \%)$, accompanied by $196 \mathrm{mg}(25 \%)$ of the mono-substituted material. ${ }^{1} \mathrm{H} \mathrm{NMR}\left(\mathrm{CDCl}_{3}, 300 \mathrm{MHz}\right): \delta$ $7.16\left(\mathrm{t},{ }^{3} J_{\mathrm{HH}}=7.7 \mathrm{~Hz}, 1 \mathrm{H}, \operatorname{Ar} H\right), 6.54-6.44(\mathrm{~m}, 3 \mathrm{H}, \mathrm{Ar} H), 5.97-5.82(\mathrm{~m}, 2 \mathrm{H}, \mathrm{CH}), 5.22-5.06(\mathrm{~m}$, $\left.4 \mathrm{H},=\mathrm{CH}_{2}\right), 4.02\left(\mathrm{t},{ }^{3} J_{\mathrm{HH}}=6.8 \mathrm{~Hz}, 4 \mathrm{H}, \mathrm{OCH}_{2}\right), 2.60-2.50\left(\mathrm{~m}, 4 \mathrm{H}, \mathrm{CH}_{2}\right) \cdot{ }^{13} \mathrm{C}\left\{{ }^{1} \mathrm{H}\right\} \mathrm{NMR}\left(\mathrm{CDCl}_{3}\right.$, $75 \mathrm{MHz}): \delta$ 160.1, 134.4, 129.8, 117.0, 106.8, 101.5, 67.1, 33.6. IR $v\left(\mathrm{~cm}^{-1}\right) 3081(\mathrm{~s}), 2926(\mathrm{~s})$, $2874(\mathrm{~s}), 1642$ (s), 1601 (s), 1492 (s), 1465 (s), 1383 (m), 1333 (m), 1264 (s), 1181 (s), 1154 (s), 
1045 (s), 992 (s), 916 (s), 835 (s), 762 (s), 686 (s). HR-MS (+EI): Calc'd for $\mathrm{C}_{14} \mathrm{H}_{18} \mathrm{O}_{2}, \mathrm{M}^{+\bullet}, \mathrm{m} / \mathrm{z}$ 218.1307. Found, $m / z 218.1319$.

\subsection{Preparation and characterization of RCM products.}

\section{Preparation of 7 a.}

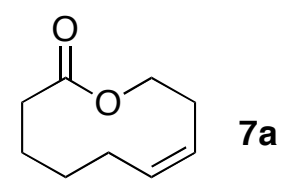

A solution of catalyst $1(43 \mathrm{mg}, 0.0508 \mathrm{mmol})$ in $10 \mathrm{~mL} \mathrm{CH}_{2} \mathrm{Cl}_{2}$ was added all at once to a solution of $6 \mathbf{a}(195 \mathrm{mg}, 1.07 \mathrm{mmol})$ in $400 \mathrm{~mL} \mathrm{CH}_{2} \mathrm{Cl}_{2}$. The solution was stirred at $22{ }^{\circ} \mathrm{C}$ for 15 min, then heated to reflux for $5 \mathrm{~h}$. After this time, the solution was concentrated to ca. $1 \mathrm{~mL}$ and the product purified by chromatography on silica gel (10\% EtOAc in hexanes). Multiple cycles of chromatography were required to remove traces of $\mathrm{Ru}$ and obtain pure $\mathbf{7 a}$, as a colourless oil suitable for constructing $\mathrm{GC}$ calibration curves. Isolated yield: $99 \mathrm{mg}(60 \%) .{ }^{1} \mathrm{H} \mathrm{NMR}\left(\mathrm{CDCl}_{3}\right.$, $300 \mathrm{MHz}): \delta 5.51-5.44(\mathrm{~m}, 2 \mathrm{H}, \mathrm{CH}), 4.19-4.07\left(\mathrm{~m}, 2 \mathrm{H}, \mathrm{OCH}_{2}\right), 2.40-2.22\left(\mathrm{~m}, 4 \mathrm{H}, \mathrm{CH}_{2} \mathrm{CO}\right.$ and $\left.\mathrm{CH}_{2}\right), 2.09-1.95\left(\mathrm{~m}, 2 \mathrm{H}, \mathrm{CH}_{2}\right), 1.82-1.72\left(\mathrm{~m}, 2 \mathrm{H}, \mathrm{CH}_{2}\right), 1.69-1.55\left(\mathrm{~m}, 2 \mathrm{H}, \mathrm{CH}_{2}\right) .{ }^{13} \mathrm{C}\left\{{ }^{1} \mathrm{H}\right\} \mathrm{NMR}$ $\left(\mathrm{CDCl}_{3}, 125 \mathrm{MHz}\right): \delta 174.8,134.4,124.7,61.7,35.5,28.6,25.2,24.8,22.9 . \operatorname{IR} v\left(\mathrm{~cm}^{-1}\right) 2961(\mathrm{~s})$, 2931 (s), 2857 (s), 1732 (s), 1596 (w), 1459 (w), 1448 (w), 1382 (w), 1256 (m), $1182(\mathrm{~m}), 1157$ (m), 1079 (m), 1024 (m), 968 (w), 799 (w). HR-MS (+EI): Calc'd for $\mathrm{C}_{9} \mathrm{H}_{14} \mathrm{O}_{2}, \mathrm{M}^{+\bullet}, \mathrm{m} / \mathrm{z}$ 154.0994. Found, $m / z 154.0997$. 


\section{Preparation of $7 \mathrm{c}$.}

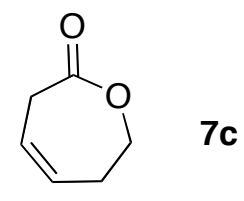

The reaction was carried out as for 7a. The product was much more easily purified, one cycle of chromatography (silica gel, 10\% diethyl ether in pentane) being sufficient to yield a colourless oil. Isolated yield: $71 \mathrm{mg}(60 \%) . \quad{ }^{1} \mathrm{H} \mathrm{NMR}\left(\mathrm{CDCl}_{3}, 300 \mathrm{MHz}\right): \delta 5.75-5.64(\mathrm{~m}, 1 \mathrm{H}, \mathrm{CH}), 5.57-$ $5.45(\mathrm{~m}, 1 \mathrm{H}, \mathrm{CH}), 4.39\left(\mathrm{t},{ }^{3} J_{\mathrm{HH}}=5.6 \mathrm{~Hz}, 2 \mathrm{H}, \mathrm{OCH}_{2}\right), 3.42-3.30\left(\mathrm{~m}, 2 \mathrm{H}, \mathrm{CH}_{2}\right), 2.53-2.42(\mathrm{~m}, 2 \mathrm{H}$, $\left.\mathrm{CH}_{2}\right) ;{ }^{13} \mathrm{C}\left\{{ }^{1} \mathrm{H}\right\}$ NMR $\left(\mathrm{CDCl}_{3}, 125 \mathrm{MHz}\right): \delta$ 173.5, 129.3, 118.8, 65.8, 33.7 30.2. IR $v\left(\mathrm{~cm}^{-1}\right) 3027$ (m), $2962(\mathrm{~m}), 2894(\mathrm{~m}), 1735(\mathrm{~s}), 1662(\mathrm{~m}), 1481(\mathrm{w}), 1425(\mathrm{w}), 1384(\mathrm{w}), 1292(\mathrm{~m}), 1252(\mathrm{w})$, 1167 (m), 1083 (w), 1054 (m), 1026 (m), 970 (w), 853 (w), 688 (m). HR-MS (+EI): Calc'd for $\mathrm{C}_{6} \mathrm{H}_{8} \mathrm{O}_{2}, \mathrm{M}^{+*}, m / z$ 112.0524. Found, $m / z$ 112.0534.

\section{Preparation of 9a.}

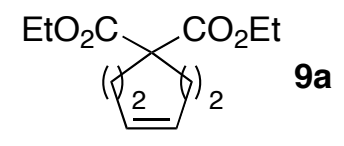

To a solution of $8 \mathbf{a}(51 \mathrm{mg}, 0.19 \mathrm{mmol})$ in $37 \mathrm{~mL} \mathrm{CH}_{2} \mathrm{Cl}_{2}$ was added $731 \mu \mathrm{L}$ of a stock solution of catalyst $1(11 \mathrm{mg} / \mathrm{mL}, 0.0095 \mathrm{mmol})$. The solution was stirred at $22{ }^{\circ} \mathrm{C}$ for $15 \mathrm{~min}$, then heated to reflux for 1 hour. After this time, the solution was concentrated to ca. $1 \mathrm{~mL}$ and the product purified by chromatography (silica gel, 10\% EtOAc in hexanes). One cycle of chromatography was sufficient to separate 9a from spent Ru, yielding the RCM product as a colourless oil. Isolated yield: $21 \mathrm{mg}(47 \%) .{ }^{1} \mathrm{H} \mathrm{NMR}\left(\mathrm{CDCl}_{3}, 300 \mathrm{MHz}\right): \delta 5.66(\mathrm{~s}, 2 \mathrm{H}, \mathrm{CH}), 4.18\left(\mathrm{q},{ }^{3} \mathrm{~J}_{\mathrm{HH}}=\right.$ $\left.6.9 \mathrm{~Hz}, 4 \mathrm{H}, \mathrm{OCH}_{2}\right), 2.21\left(\mathrm{~s}, 8 \mathrm{H}, \mathrm{CH}_{2}\right), 1.24\left(\mathrm{t},{ }^{3} J_{\mathrm{HH}}=7.2 \mathrm{~Hz}, 6 \mathrm{H}, \mathrm{CH}_{3}\right) .{ }^{13} \mathrm{C}\left\{{ }^{1} \mathrm{H}\right\} \mathrm{NMR}\left(\mathrm{CDCl}_{3}\right.$, 75 MHz): $\delta$ 172.3, 130.8, 61.1, 57.9, 31.9, 24.4, 14.0. IR $v\left(\mathrm{~cm}^{-1}\right) 2980(\mathrm{~m}), 2935$ (m), $1729(\mathrm{~s})$, 1447 (m), 1366 (m), 1294 (m), 1242 (s), 1219 (s), 1083 (m), 1048 (m), 871 (w), 803 (w). HRMS (+EI): Calc'd for $\mathrm{C}_{11} \mathrm{H}_{15} \mathrm{O}_{3}$, [M- $\left.\mathrm{C}_{2} \mathrm{H}_{5} \mathrm{O}\right]^{+\bullet}, m / z$ 195.1021. Found, $m / z$ 195.1026. 
Preparation of 11a.

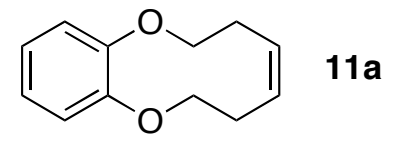

A solution of catalyst $1(20 \mathrm{mg}, 0.02 \mathrm{mmol})$ in $10 \mathrm{~mL} \mathrm{CH} \mathrm{Cl}_{2}$ was added all at once to a solution of $10 \mathbf{a}(100 \mathrm{mg}, 0.45 \mathrm{mmol})$ in $400 \mathrm{~mL} \mathrm{CH}_{2} \mathrm{Cl}_{2}$. The solution was stirred at $22{ }^{\circ} \mathrm{C}$ for $15 \mathrm{~min}$, then heated to reflux as for 9a. Multiple cycles of chromatography (silica gel, 10\% EtOAc in hexanes) were required to remove traces of $\mathrm{Ru}$, and obtain $\mathbf{1 1 a}$ as a colourless oil. Isolated yield: $27 \mathrm{mg}(32 \%) .{ }^{1} \mathrm{H} \mathrm{NMR}\left(\mathrm{CDCl}_{3}, 300 \mathrm{MHz}\right): \delta$ 6.94-6.87 (m, 4H, $\left.\mathrm{ArH}\right), 5.78-5.70(\mathrm{~m}, 2 \mathrm{H}, \mathrm{CH})$, $4.02\left(\mathrm{t},{ }^{3} J_{\mathrm{HH}}=6.02 \mathrm{~Hz}, 4 \mathrm{H}, \mathrm{OCH}_{2}\right), 2.59-2.49\left(\mathrm{~m}, 4 \mathrm{H}, \mathrm{CH}_{2}\right) .{ }^{13} \mathrm{C}\left\{{ }^{1} \mathrm{H}\right\} \mathrm{NMR}\left(\mathrm{CDCl}_{3}, 125 \mathrm{MHz}\right)$ : $\delta$ 149.6, 128.7, 121.7, 115.7, 69.2, 33.1. IR v ( $\left.\mathrm{cm}^{-1}\right) 2925(\mathrm{~s}), 2921(\mathrm{~s}), 2853(\mathrm{~m}), 1735(\mathrm{~m}), 1724$ (m), $1591(\mathrm{~m}), 1492$ (m), $1466(\mathrm{~m}), 1378$ (w), 1253 (s), $1120(\mathrm{~m}), 1070(\mathrm{w}), 1040$ (w), 797 (w), 747 (m). HR-MS (+EI): Calc'd for $\mathrm{C}_{12} \mathrm{H}_{14} \mathrm{O}_{2}, \mathrm{M}^{+\bullet}, m / z$ 190.0994. Found, $m / z$ 190.0991. 


\subsection{NMR Spectra.}

${ }^{1} \mathrm{H}$ NMR Spectrum of $6 \mathrm{a}\left(500 \mathrm{MHz}, \mathrm{CDCl}_{3}\right)$.

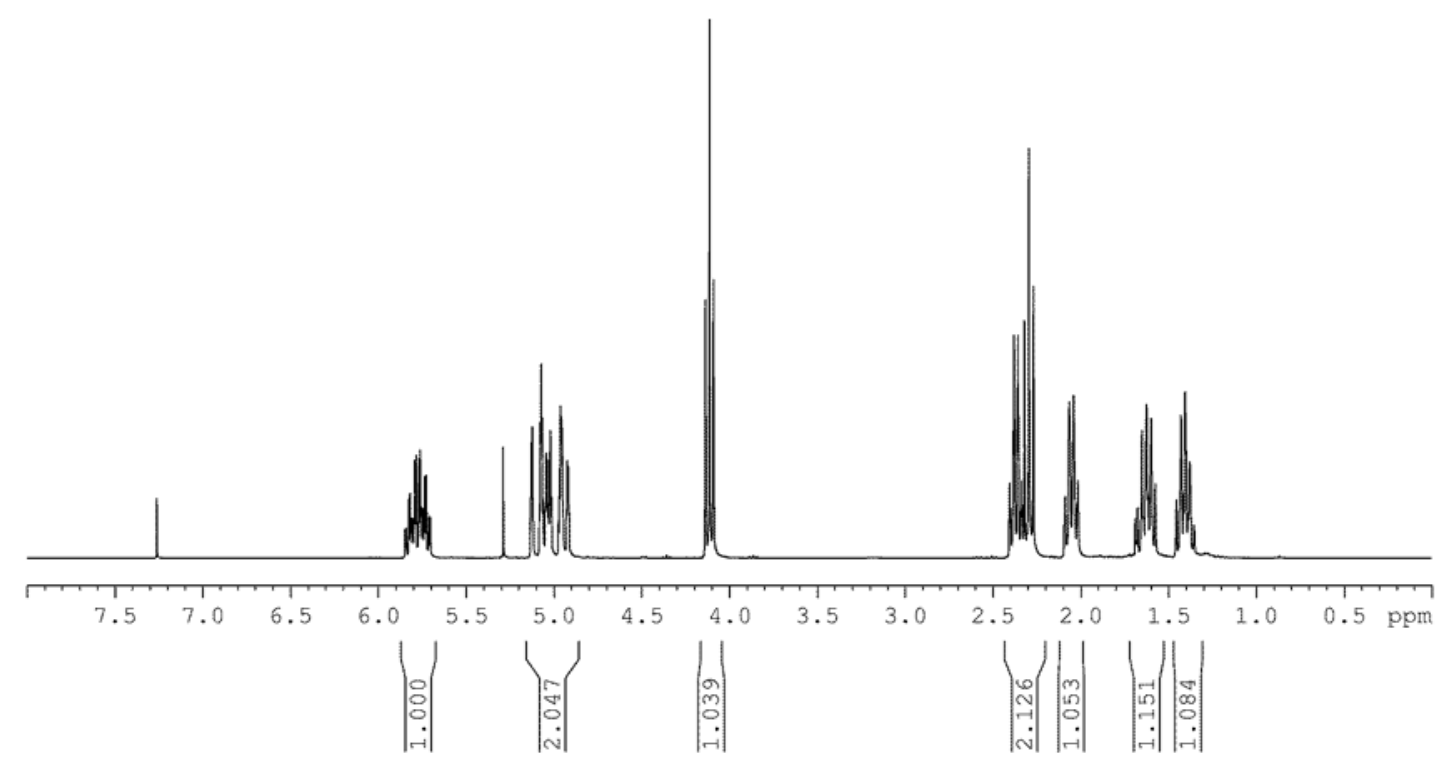

${ }^{13} \mathrm{C}\left\{{ }^{1} \mathrm{H}\right\}$ NMR Spectrum of $6 \mathrm{a}\left(125 \mathrm{MHz}, \mathrm{CDCl}_{3}\right)$.

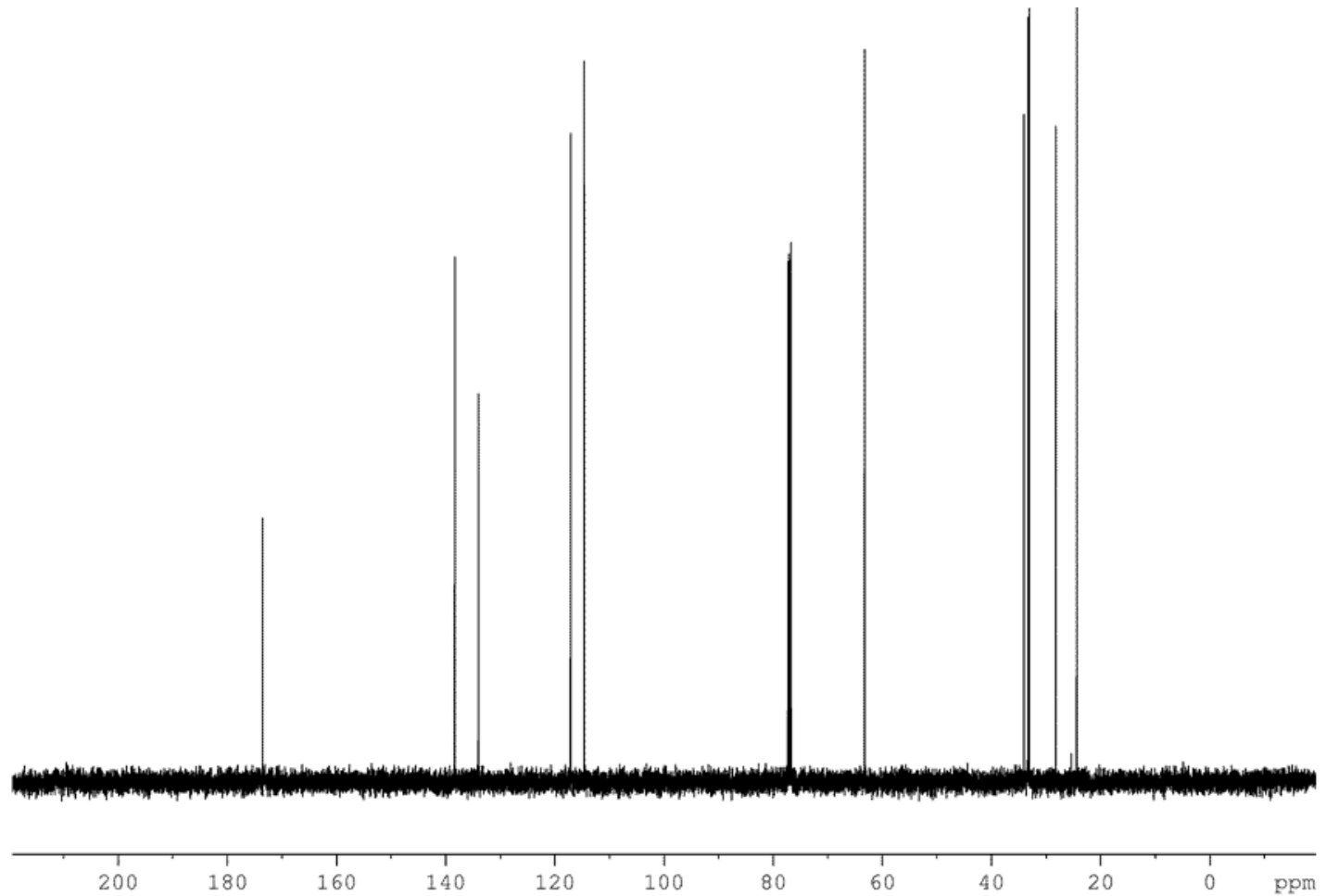


${ }^{1} \mathrm{H}$ NMR spectrum of $6 \mathrm{~b}\left(500 \mathrm{MHz}, \mathrm{CDCl}_{3}\right)$.

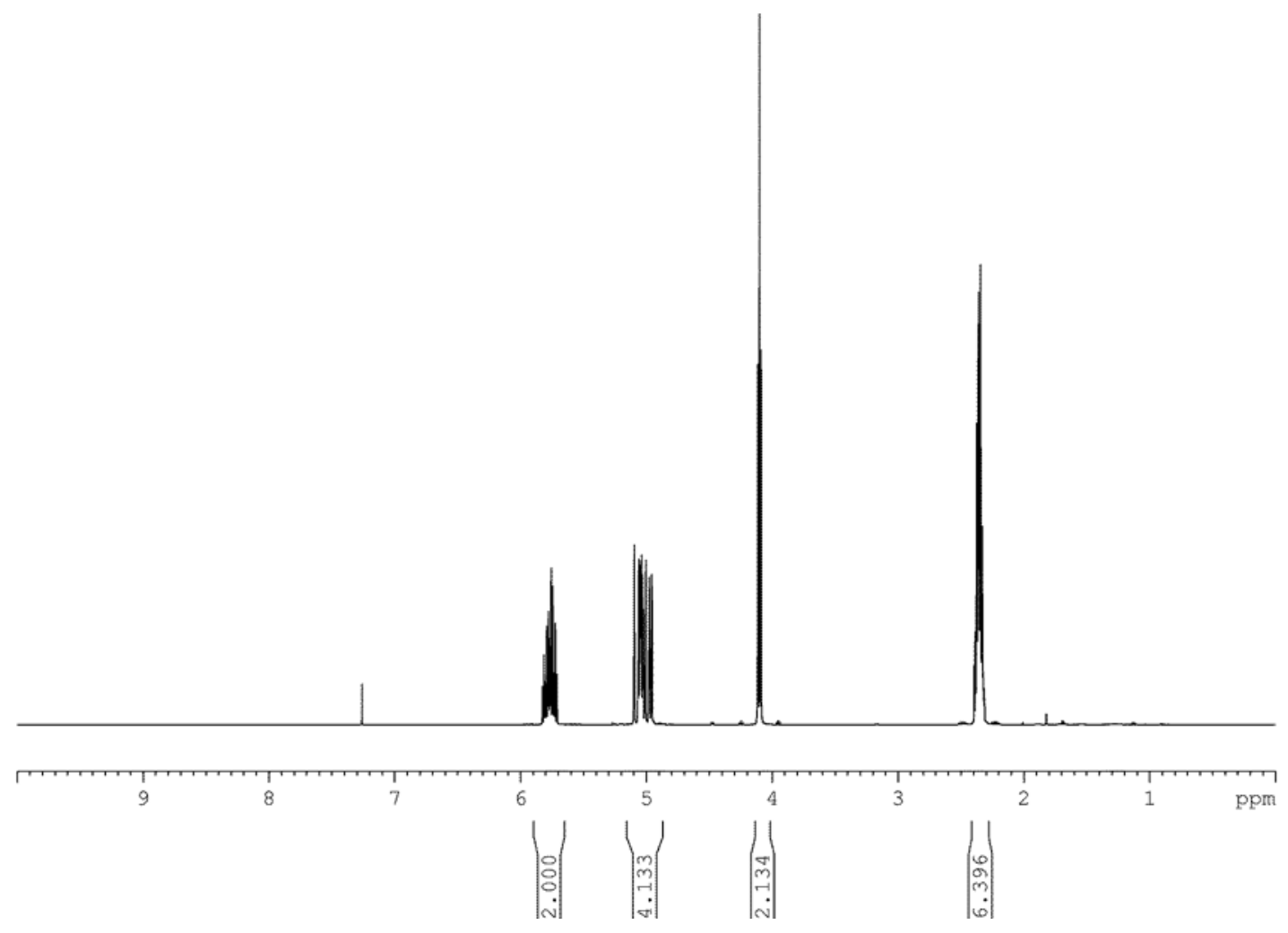

${ }^{13} \mathrm{C}\left\{{ }^{1} \mathrm{H}\right\}$ NMR spectrum of $6 \mathrm{~b}\left(125 \mathrm{MHz}, \mathrm{CDCl}_{3}\right)$.

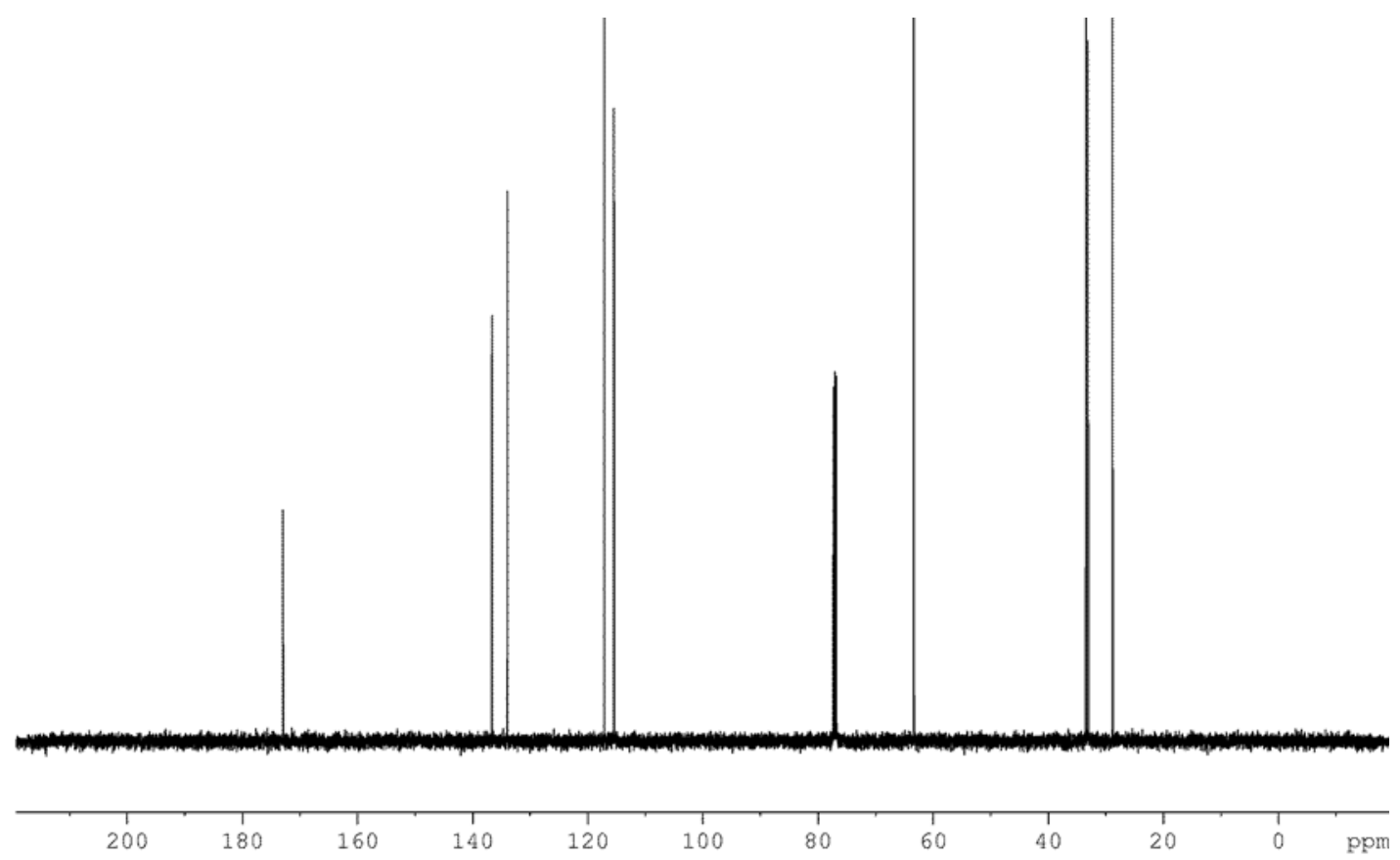


${ }^{1} \mathrm{H}$ NMR Spectrum of $6 \mathrm{c}\left(300 \mathrm{MHz}, \mathrm{CDCl}_{3}\right)$.

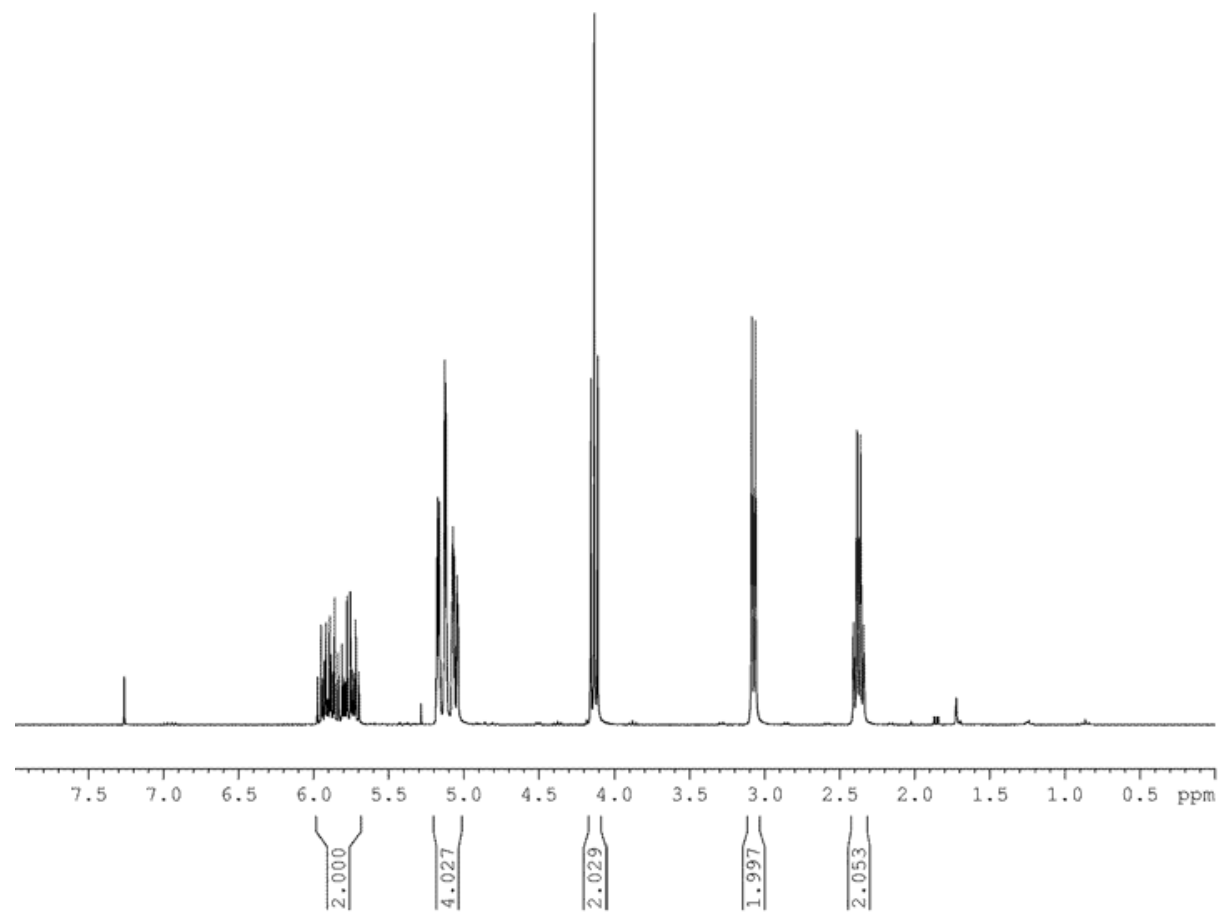

${ }^{13} \mathrm{C}\left\{{ }^{1} \mathrm{H}\right\}$ NMR Spectrum of $6 \mathrm{c}\left(75 \mathrm{MHz}, \mathrm{CDCl}_{3}\right)$.

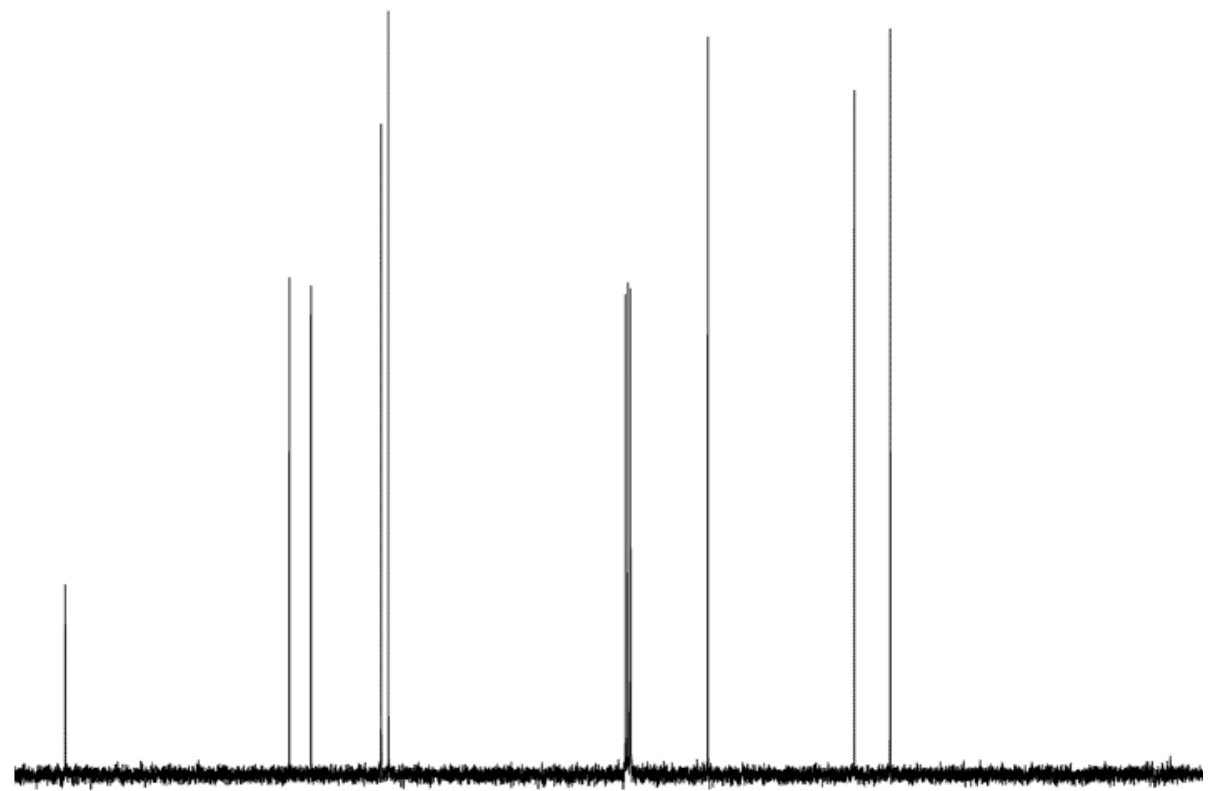

$\begin{array}{lllllllllllllllllll}170 & 160 & 150 & 140 & 130 & 120 & 110 & 100 & 90 & 80 & 70 & 60 & 50 & 40 & 30 & 20 & 10 & 0 & \mathrm{ppm}\end{array}$ 
${ }^{1} \mathrm{H}$ NMR spectrum of $7 \mathrm{a}\left(300 \mathrm{MHz}, \mathrm{CDCl}_{3}\right)$.

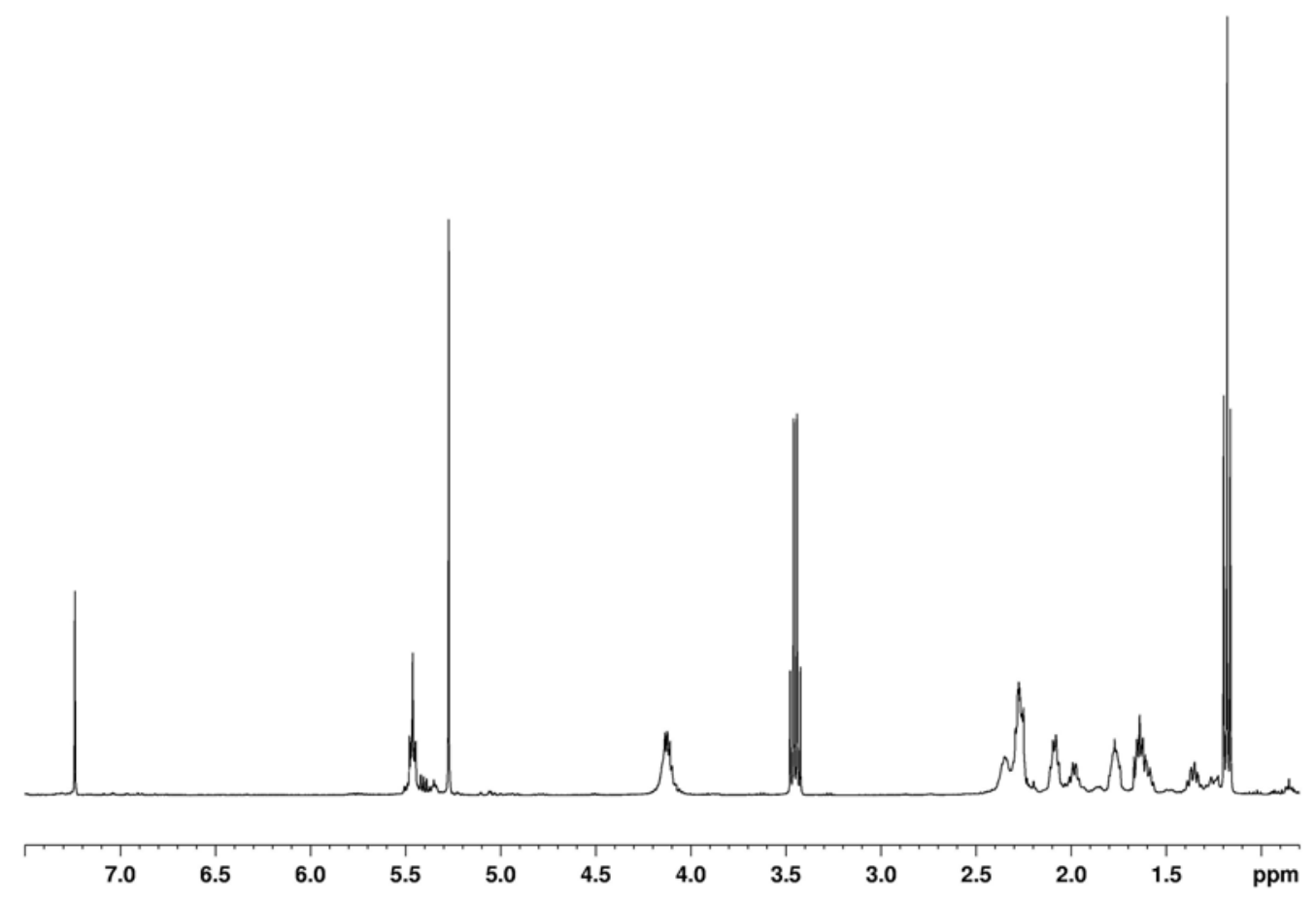

${ }^{13} \mathrm{C}\left\{{ }^{1} \mathrm{H}\right\}$ spectrum of $7 \mathrm{a}\left(75 \mathrm{MHz}, \mathrm{CDCl}_{3}\right)$.

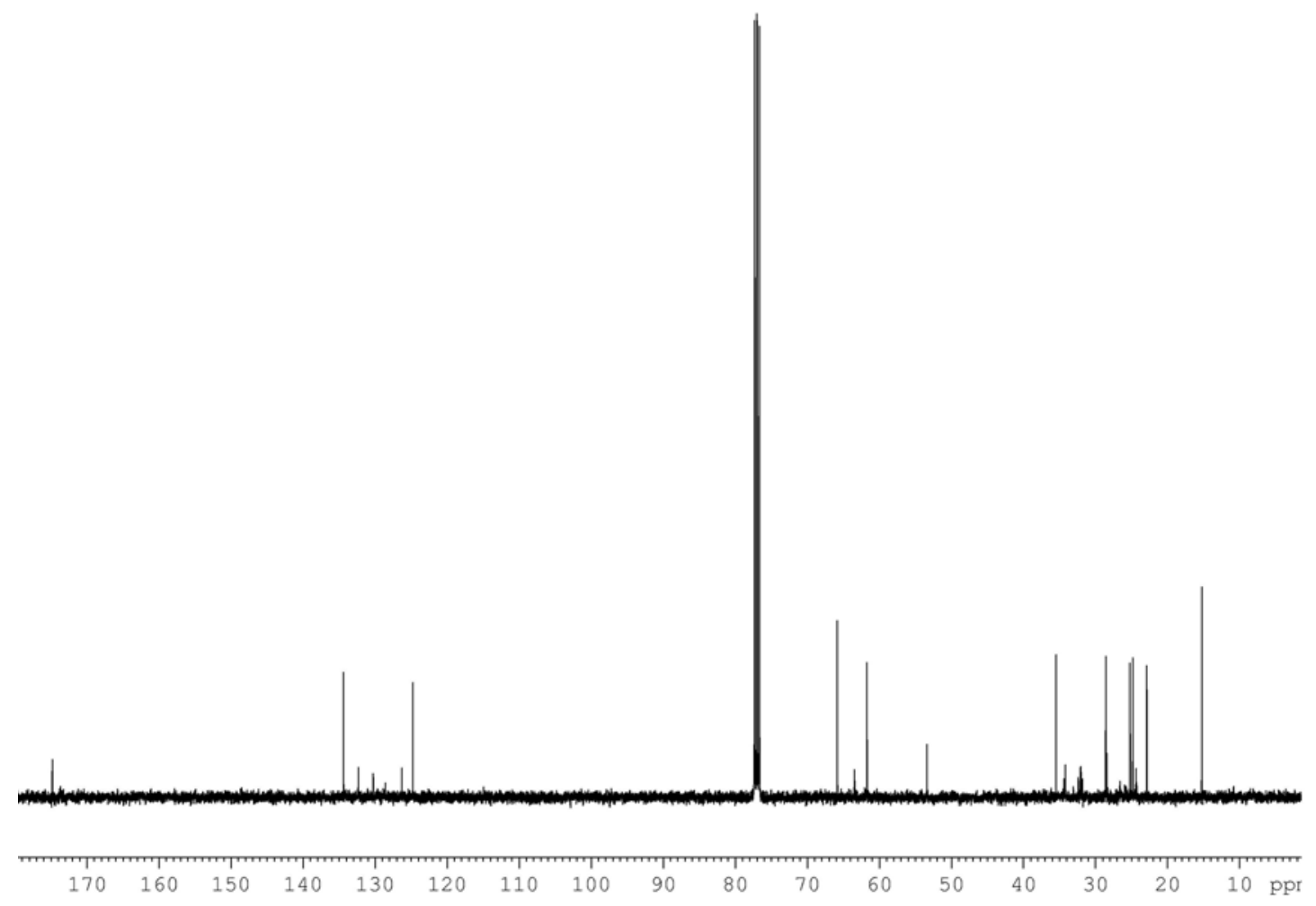


${ }^{1} \mathrm{H}$ NMR spectrum of $7 \mathrm{c}\left(300 \mathrm{MHz}, \mathrm{CDCl}_{3}\right)$.

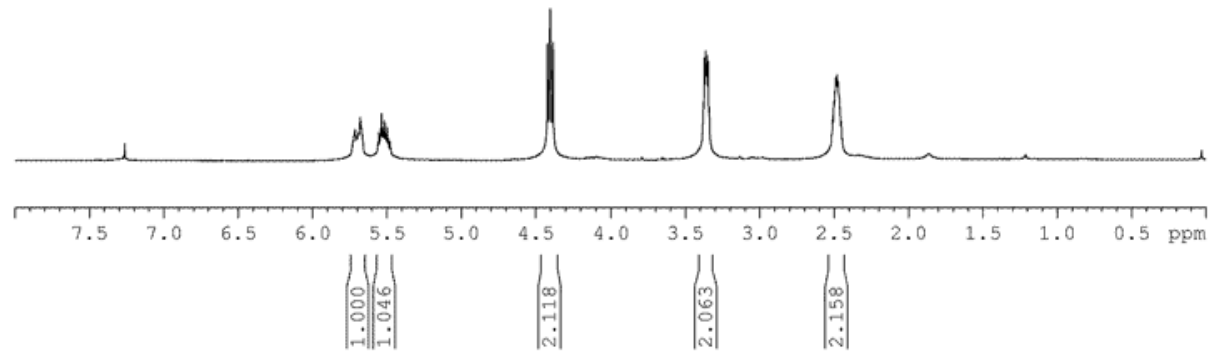

${ }^{13} \mathrm{C}\left\{{ }^{1} \mathrm{H}\right\}$ NMR spectrum of $7 \mathrm{c}\left(75 \mathrm{MHz}, \mathrm{CDCl}_{3}\right)$.

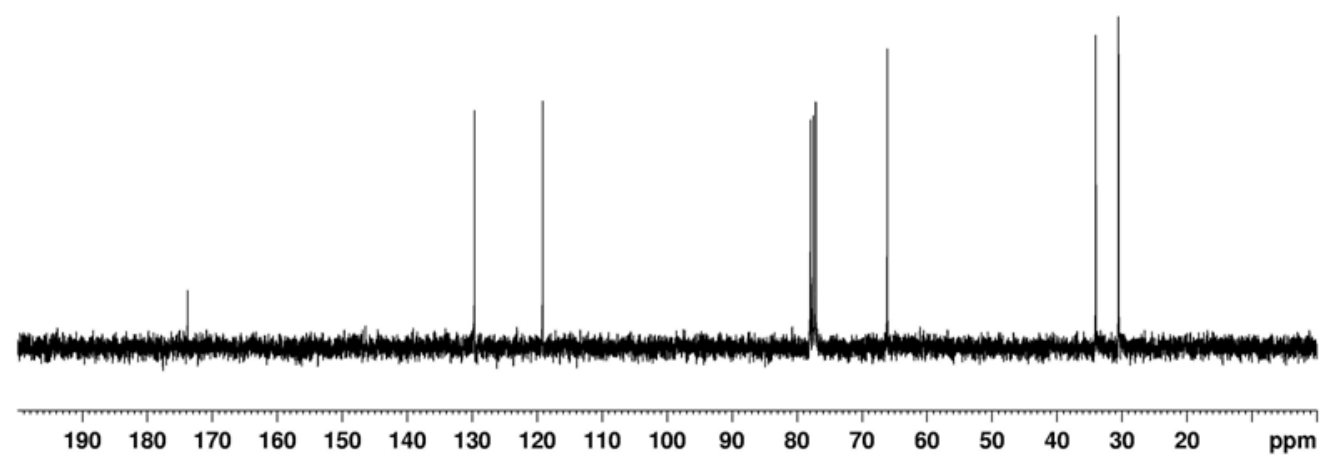


${ }^{1} \mathrm{H}$ NMR Spectrum of $8 \mathrm{a}\left(300 \mathrm{MHz}, \mathrm{CDCl}_{3}\right)$.

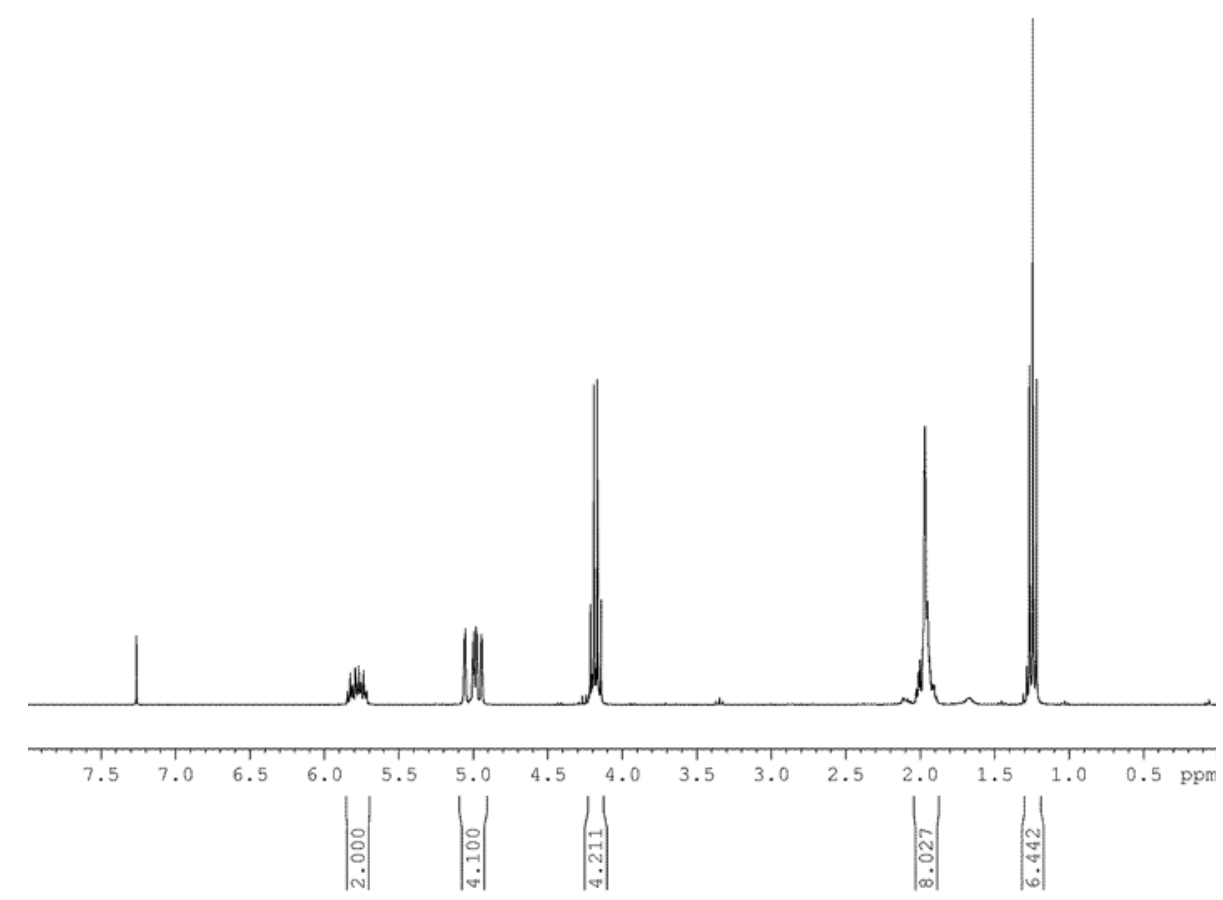

${ }^{13} \mathrm{C}\left\{{ }^{1} \mathrm{H}\right\}$ NMR Spectrum of $8 \mathrm{a}\left(75 \mathrm{MHz}, \mathrm{CDCl}_{3}\right)$.

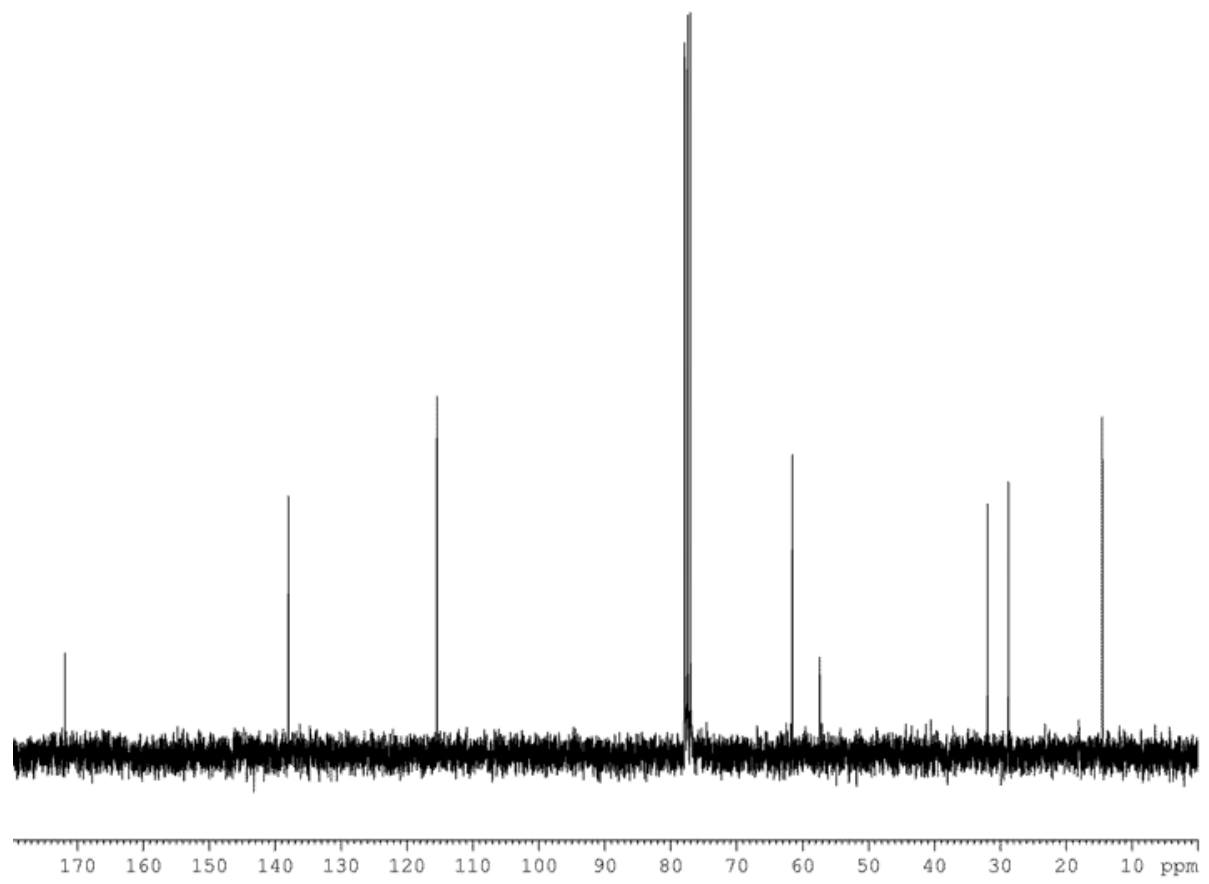


${ }^{1} \mathrm{H}$ NMR Spectrum of $9 \mathrm{a}\left(300 \mathrm{MHz}, \mathrm{CDCl}_{3}\right)$.

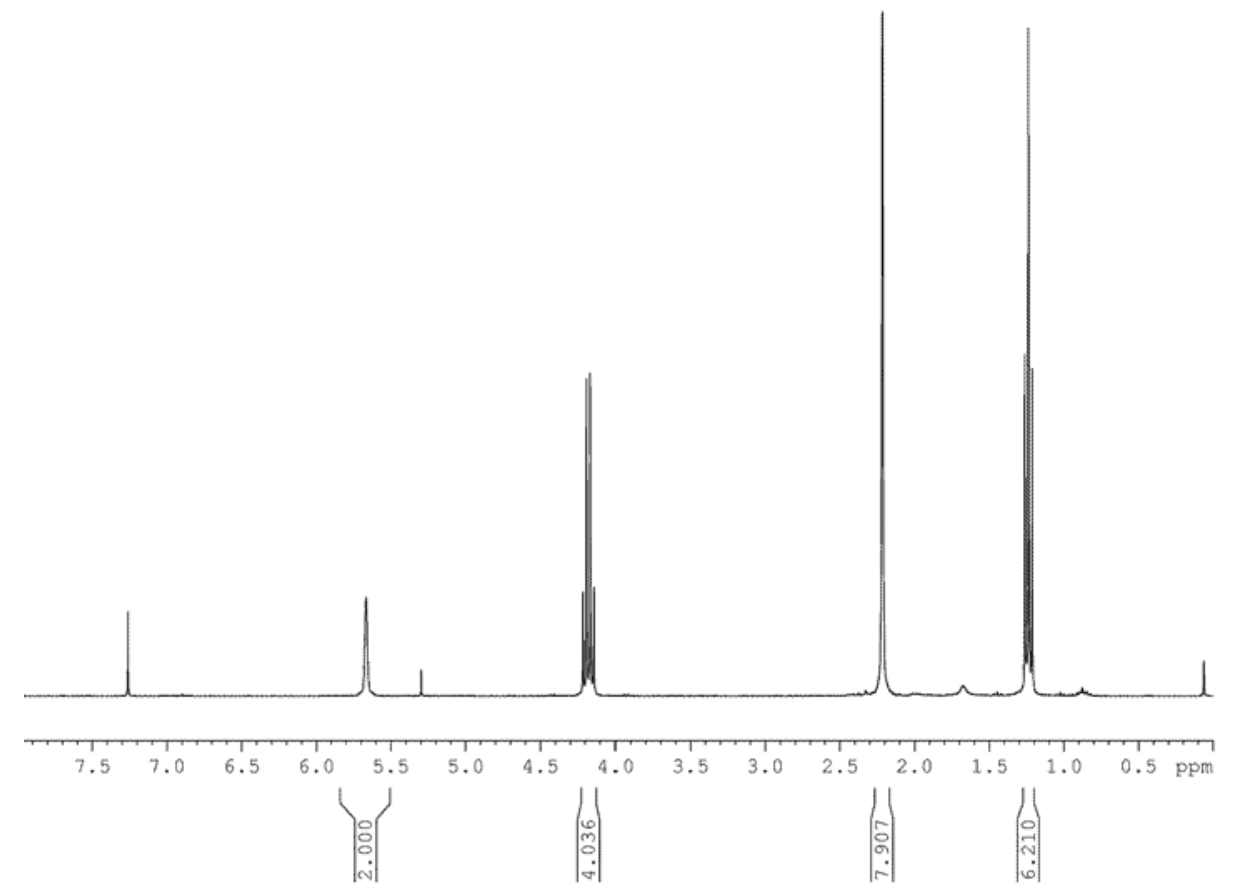

${ }^{13} \mathrm{C}\left\{{ }^{1} \mathrm{H}\right\}$ NMR Spectrum of 9a $\left(75 \mathrm{MHz}, \mathrm{CDCl}_{3}\right)$.

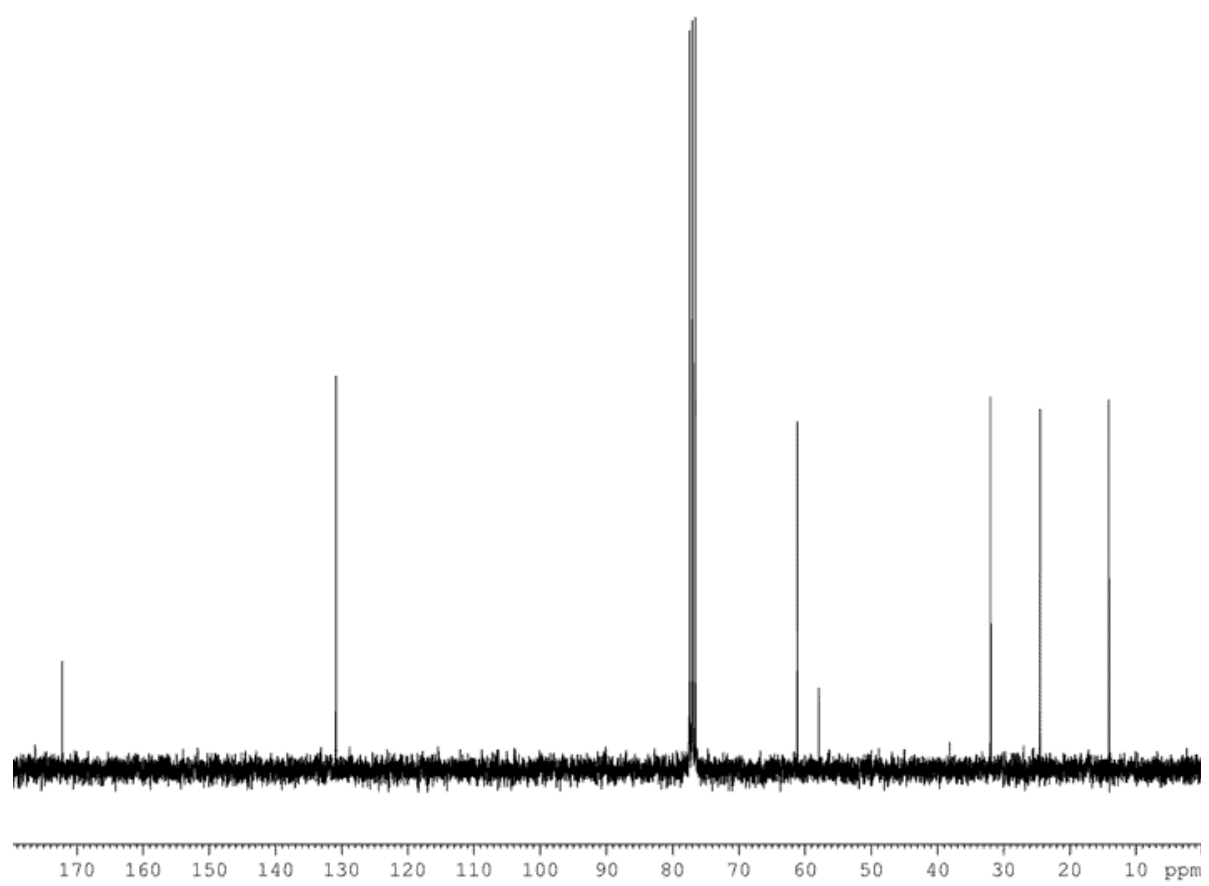


${ }^{1} \mathrm{H}$ NMR Spectrum of $10 \mathrm{a}\left(400 \mathrm{MHz}, \mathrm{CDCl}_{3}\right)$.

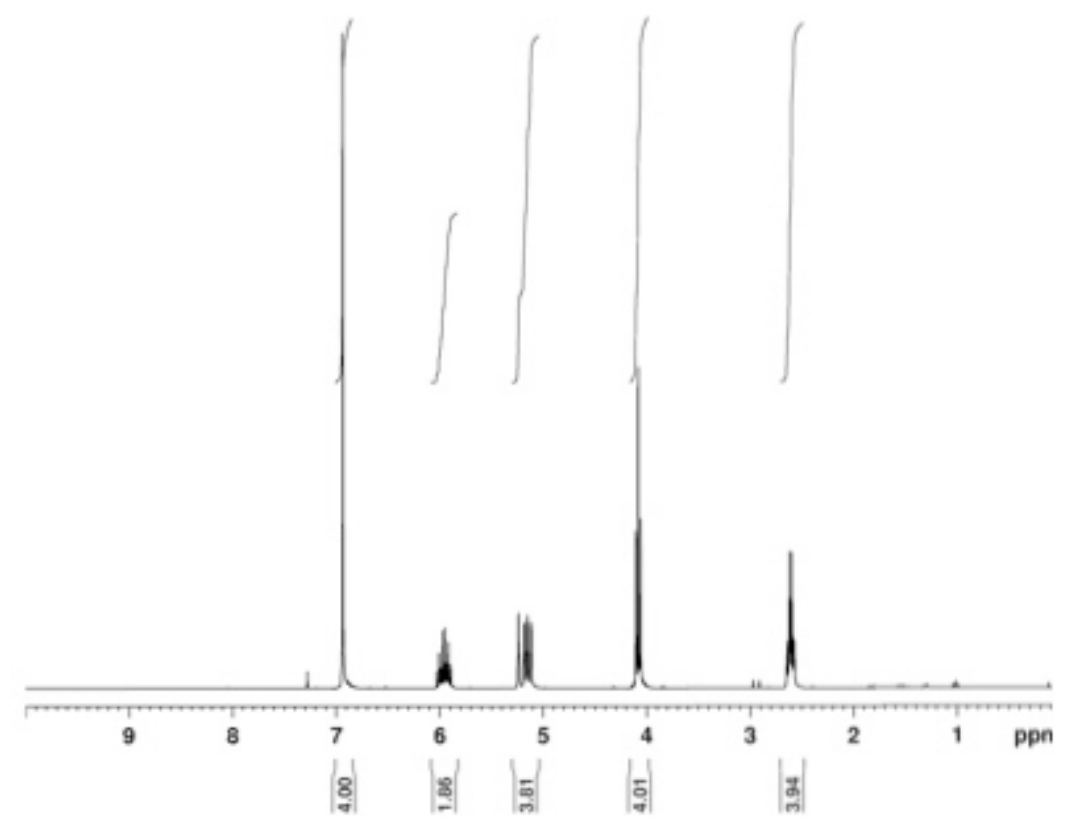

${ }^{13} \mathrm{C}\left\{{ }^{1} \mathrm{H}\right\}$ NMR Spectrum of 10a (75 $\left.\mathrm{MHz}, \mathrm{CDCl}_{3}\right)$.

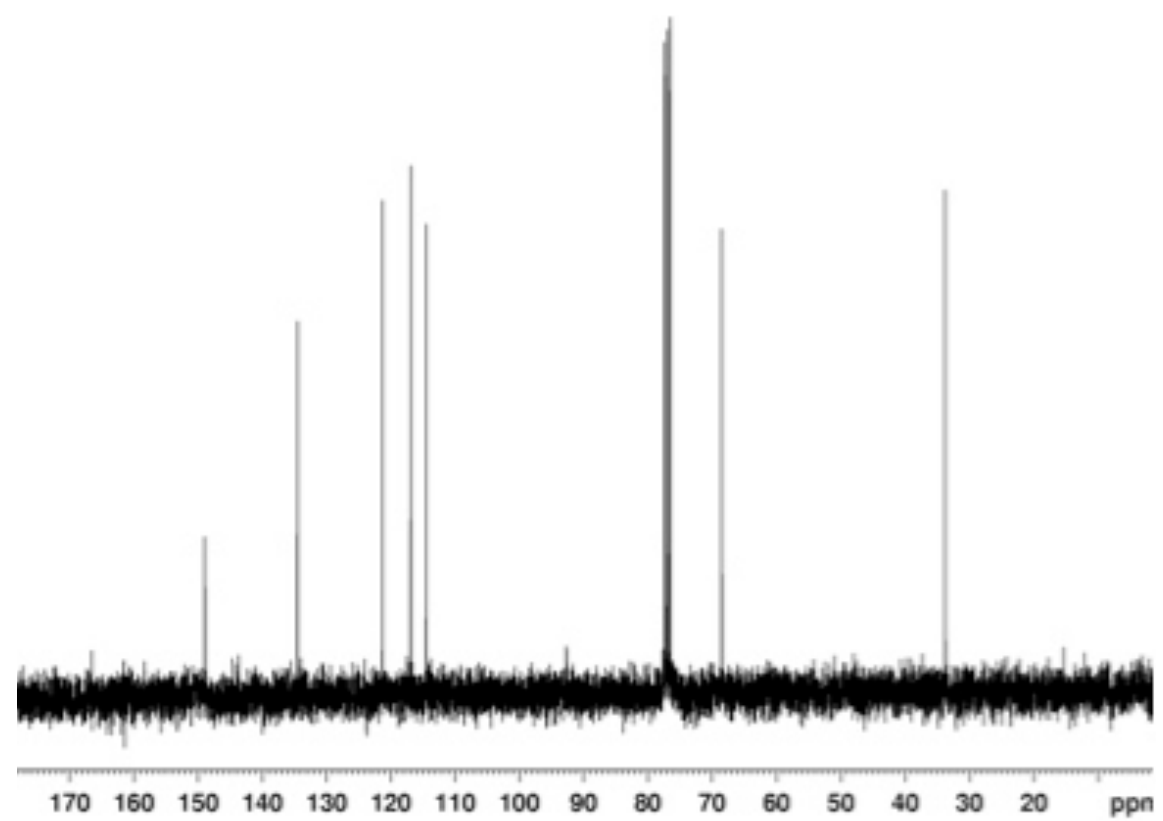


${ }^{1} \mathrm{H}$ NMR spectrum of $11 \mathrm{a}\left(300 \mathrm{MHz}, \mathrm{CDCl}_{3}\right)$.

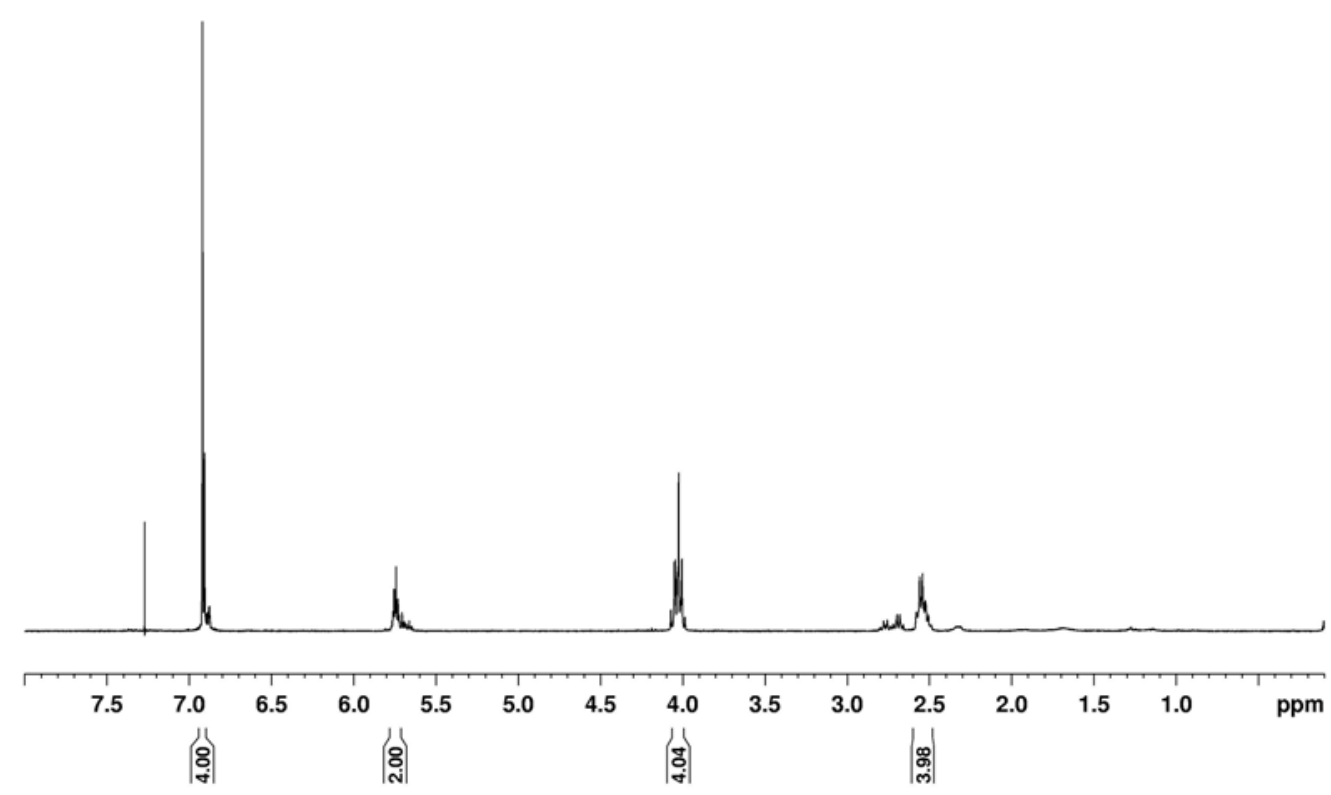

${ }^{13} \mathrm{C}\left\{{ }^{1} \mathrm{H}\right\}$ NMR spectrum of $11 \mathrm{a}\left(75 \mathrm{MHz}, \mathrm{CDCl}_{3}\right)$.

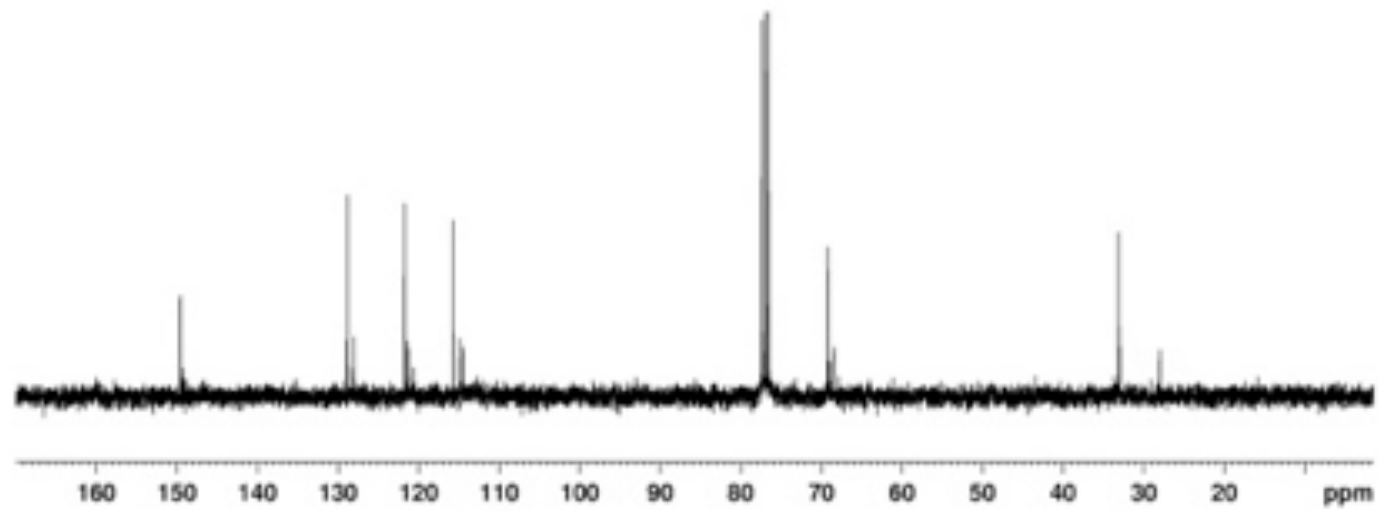


${ }^{1} \mathrm{H}$ NMR Spectrum of $12 \mathrm{a}\left(300 \mathrm{MHz}, \mathrm{CDCl}_{3}\right)$.

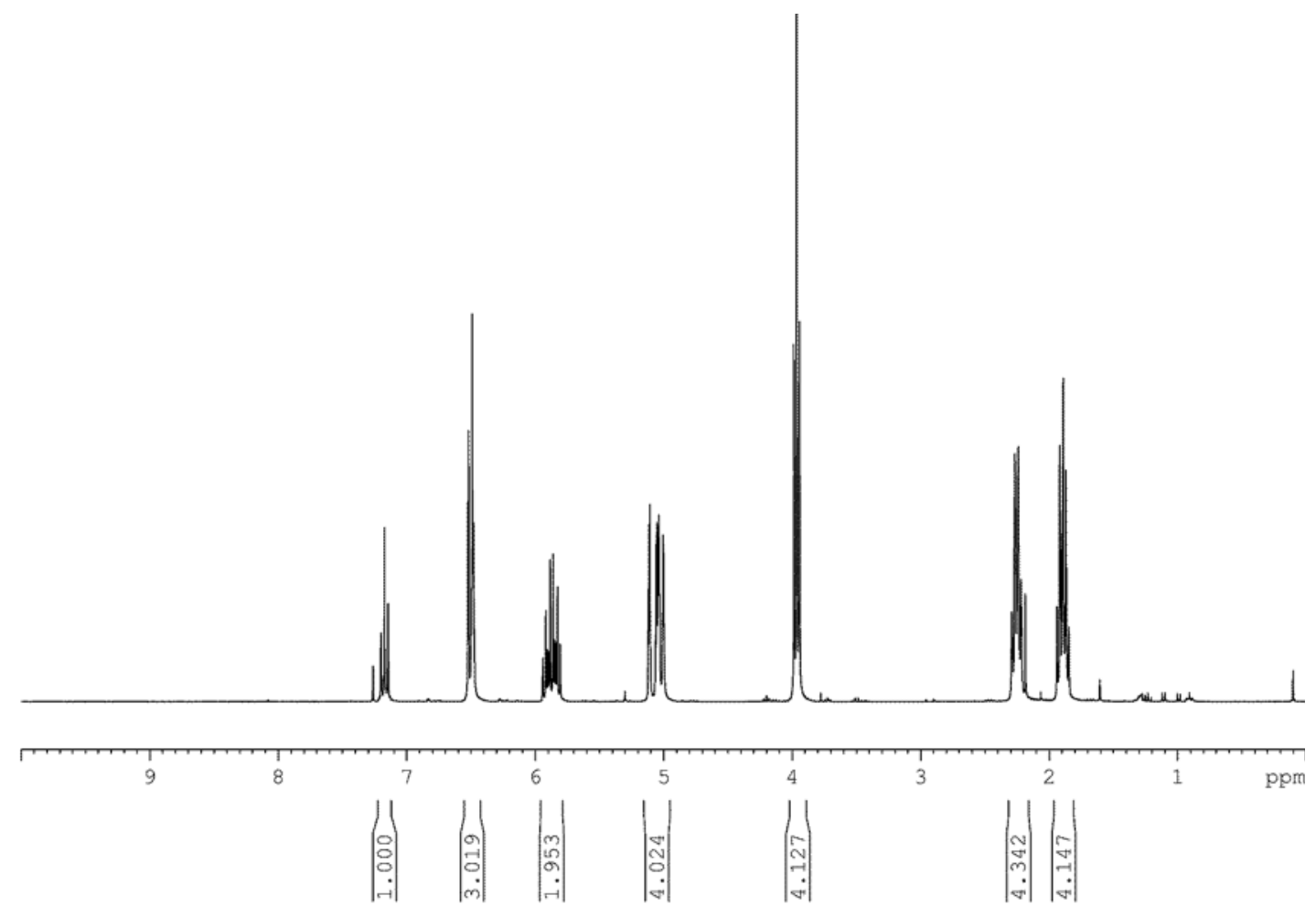

${ }^{13} \mathrm{C}\left\{{ }^{1} \mathrm{H}\right\}$ NMR Spectrum of $12 \mathrm{a}\left(75 \mathrm{MHz}, \mathrm{CDCl}_{3}\right)$.

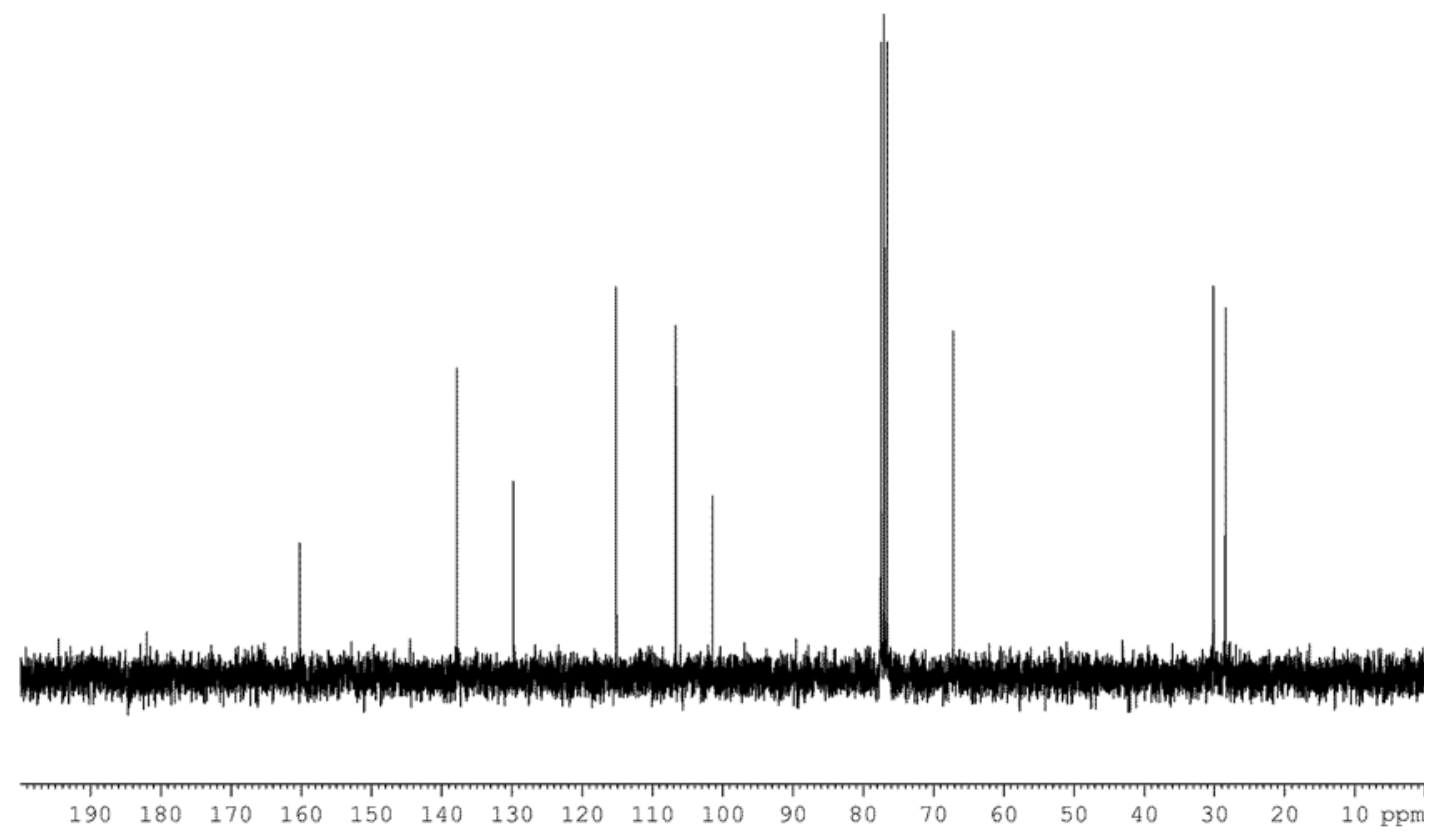


${ }^{1} \mathrm{H}$ NMR Spectrum of $12 \mathrm{~b}\left(300 \mathrm{MHz}, \mathrm{CDCl}_{3}\right)$.

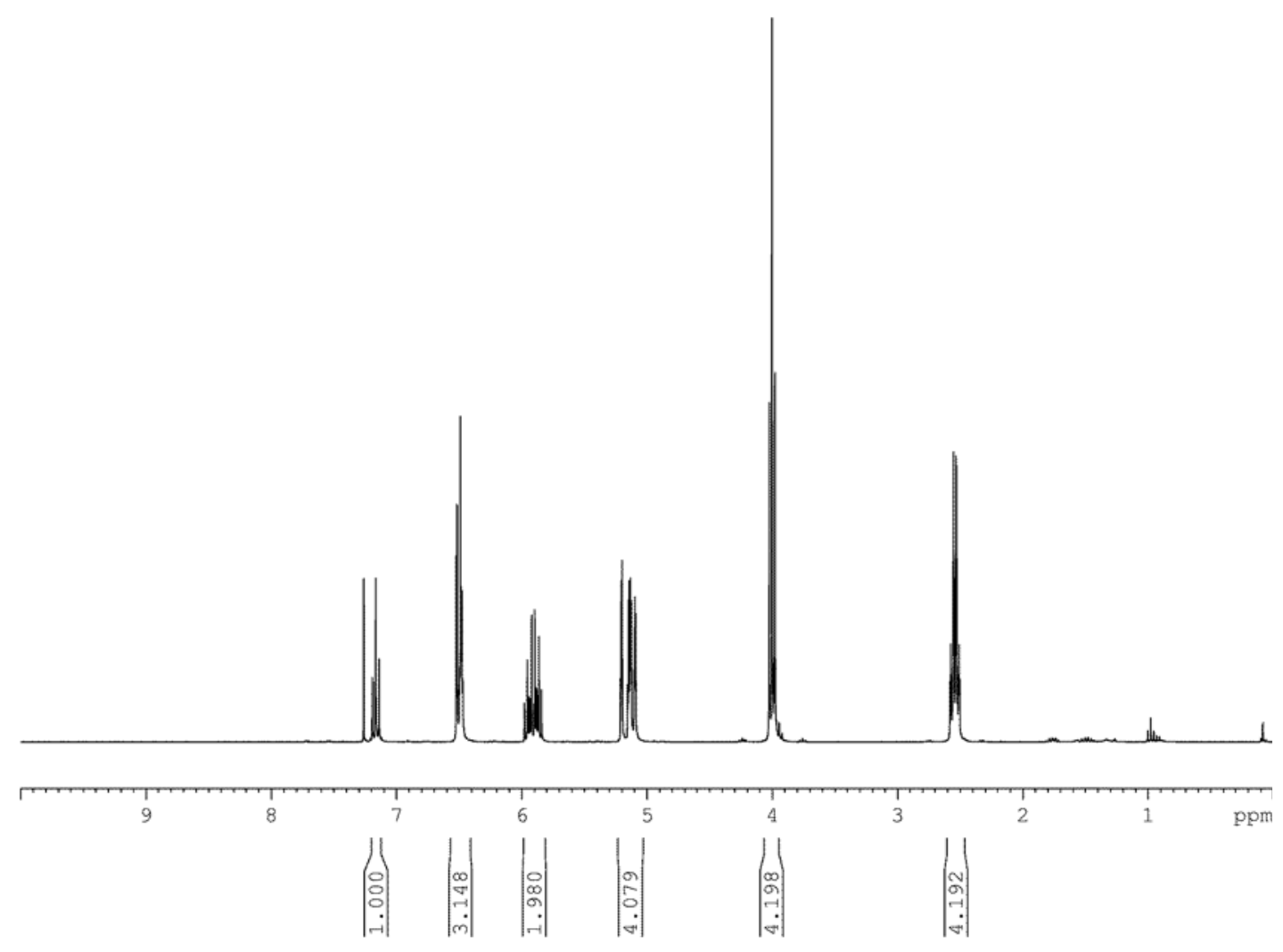

${ }^{13} \mathrm{C}\left\{{ }^{1} \mathrm{H}\right\}$ NMR Spectrum of $12 \mathrm{~b}\left(75 \mathrm{MHz}, \mathrm{CDCl}_{3}\right)$.

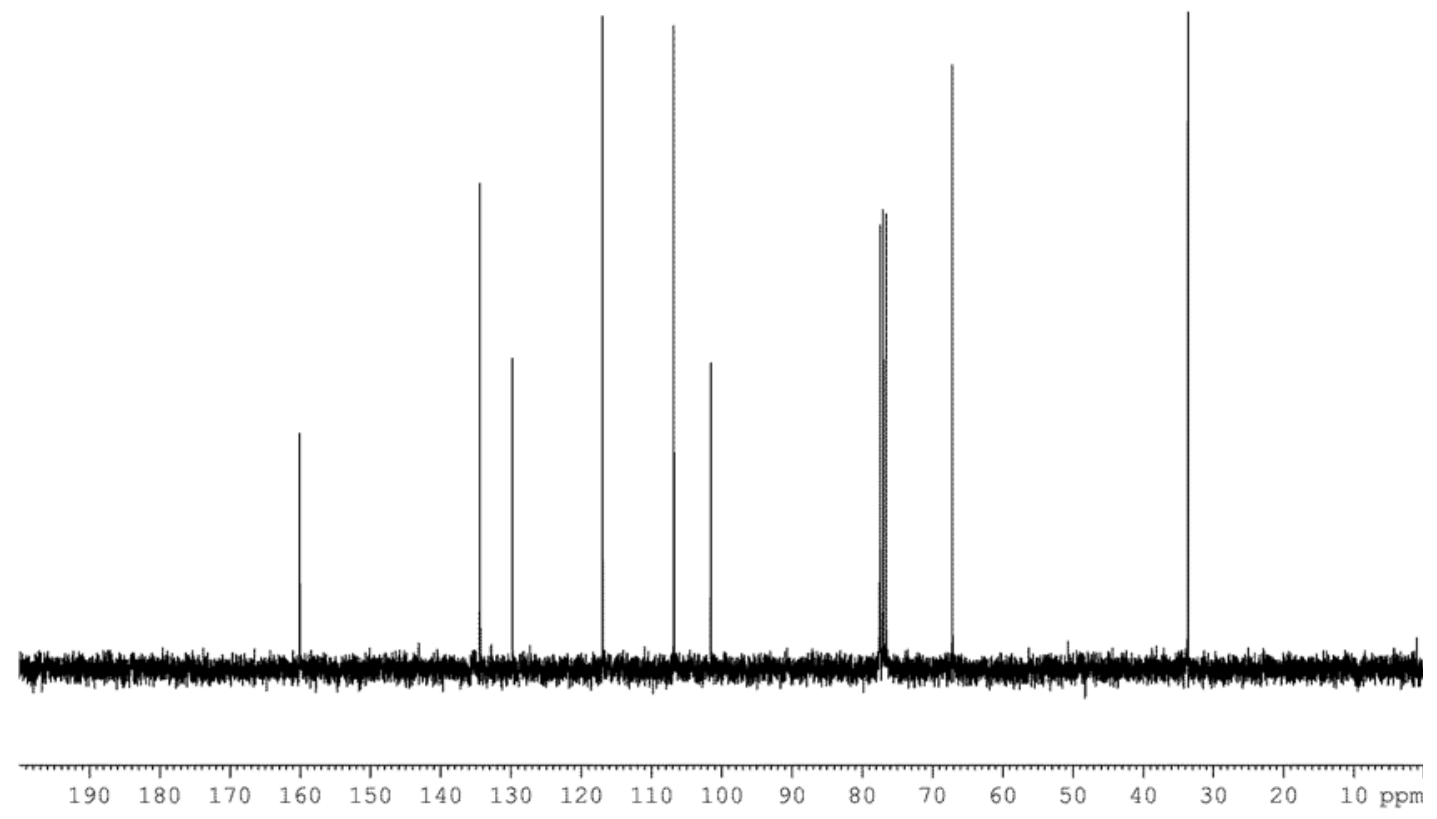




\section{References.}

(1) Scholl, M.; Trnka, T.M.; Morgan, J.P.; Grubbs, R.H. Tetrahedron Lett. 1999, 40, 2247-2250.

(2) Conrad, J.C.; Parnas, H.H.; Snelgrove, J.L.; Fogg, D.E. J. Am. Chem. Soc. 2005, 127, 11882-11883.

(3) Fürstner, A.; Langemann, K. Synthesis 1997, 792-803.

(4) Litinas, K. E.; Salteris, B. E. J. Chem. Soc., Perkin Trans. I 1997, 2869-2872.

(5) Miller, S. J.; Kim, S.-H.; Chen, Z.-R.; Grubbs, R. H. J. Am. Chem. Soc. 1995, 117, 21082109.

(6) Marsella, M. J.; Maynard, H. D.; Grubbs, R. H. Angew. Chem., Int. Ed. Engl. 1997, 36, 1101-1103.

(7) Lombardo, L. Tetrahedron Lett. 1985, 26, 381-384. 